\title{
Absence of an Optimal Capital Structure in the Famous Tradeoff Theory!
}

\author{
P.N. Brusov" ${ }^{1, *}$, T.V. Filatova ${ }^{2}$ and N.P. Orekhova ${ }^{3}$ \\ ${ }^{1}$ Applied Mathematics Department, Financial University Under the Government of Russian Federation, \\ Russia \\ ${ }^{2}$ State and Municipal Governance Department, Financial University Under the Government of Russian \\ Federation, Russia \\ ${ }^{3}$ Head of Financial and Economical Technology Department, Institute of Business, Management and Low, \\ Russia
}

\begin{abstract}
Within modern theory of capital structure and capital cost by Brusov-Filatova-Orekhova the analysis of wide known tradeoff theory has been made. It is shown that suggestion of risky debt financing (and growing credit rate near the bankruptcy) in opposite to waiting result does not lead to growing of weighted average cost of capital, WACC, which still decreases with leverage. This means the absence of minimum in the dependence of WACC on leverage as well as the absence of maximum in the dependence of company capitalization on leverage. Thus, it seems that the optimal capital structure is absent in famous tradeoff theory. The explanation to this fact has been done.
\end{abstract}

Keywords: Tradeoff theory, debt financing, company capital cost, optimal capital structure, leverage, ModiglianiMiller theory, Brusov-Filatova-Orekhova theory.

\section{INTRODUCTION}

Choosing of optimal capital structure of the company, i.e., proportion of debt and equity, which minimizes weighted average cost of capital and maximizes the company capitalization, is one of the most important tasks of financial manager and the management of a company. The search for an optimal capital structure, like the search for a "golden fleece", attracts attention of economists and financiers during many tens of years. And it is clear why: one can, nothing making, but only by changing the proportion between the values of equity capital and debt one of the company, significantly enhance the company capitalization, by other words to fulfill the primary task, to reach critical goal of the business management. Spend a little less of your own, loan slightly more (or vice versa), and company capitalization reaches a maximum.

Note, that the problem of capital structure is studied very intensively. There are theories, which consider the perfect market (Brusov et al. 2013, 2011a, 2011b, 2011c, Modigliani F., Miller M. 1958, 1963, 1966) and other ones, considering the imperfect market (Brennan M., Schwartz E. 1978, Leland H. 1994, Brennan, M. J., and E. S. Schwartz. 1984, Kane, A., A. J. Marcus, and R. L. McDonald. 1984, Dittmar A., Thakor A. 2007, Bikhchandani S., Hirshleifer D., Welch I. 1998, Post

*Address correspondence to this author at the Applied Mathematics Department, Financial University Under the Government of Russian Federation, Russia; Tel: +7-928-190-1445; E-mail: pnb1983@yahoo.com
J.E., Preston L. E., Sachs S. 2002, Filbeck G., Raymond F., Preece D. 1996, Jenter D. 2005, Baker M., Wurgler J. 2002, Graham J., Harvey C. 2001, Hovakimian A., Opler T., Titman S. 2001, Myers S., Majluf N. 1984, Myers S.C. 1984, Hovakimian A., Opler T., Titman S. 2001, Fama E., French K. 2004, Jensen M. C., Meckling W. H. 1973).

Among latter ones agent cost theory (Jensen M. C., Meckling W. H. 1973), stakeholders theory (Post J.E., Preston L. E., Sachs S. 2002), manager investment autonomy (Dittmar A., Thakor A. 2007), information cascades (Bikhchandani S., Hirshleifer D., Welch I. 1998), behavioral theories (Filbeck G., Raymond F., Preece D. 1996, Jenter D. 2005, Baker M., Wurgler J. 2002, Graham J., Harvey C. 2001, Hovakimian A., Opler T., Titman S. 2001), signaling theory (Myers S., Majluf N. 1984), pecking order theory (Myers S.C. 1984, Hovakimian A., Opler T., Titman S. 2001, Fama E., French K. 2004). Historically the conceptions on the influence of capital structure on the well-being of shareholders have developed not monotonically. We will consider the traditional (empirical) approach (Brusov et al. 2013), the Modigliani and Miller theory (Modigliani F., Miller M. 1958, 1963, 1966), tradeoff theory (Brennan M., Schwartz E. 1978, Leland H. 1994, Brennan, M. J., and E. S. Schwartz. 1984, Kane, A., A. J. Marcus, and R. L. McDonald. 1984) and modern Brusov-Filatova-Orekhova theory of capital cost and capital structure (Brusov et al. 2013, 2011a, 2011b, 2011c, 2012a, 2012b). 


\section{THE TRADITIONAL APPROACH}

The traditional (empirical) approach told to businessmen, that weighted average cost of capital, WACC, and the associated company capitalization, $V=C F / W A C C$ depend on the capital structure, the level of leverage. Debt cost always turns out to be lower, than equity cost, because first one has lower risk, because in the event of bankruptcy creditor claims are met prior to shareholders claims.

As a result an increase in the proportion of lowercost debt capital in the overall capital structure up to the limit which does not cause violation of financial sustainability and growth in risk of bankruptcy, leads to lower weighted average cost of capital, WACC.

The required by investors profitability (the equity cost) is growing, however, its growth has not led to compensation benefits from the use of more low-cost debt capital. Therefore, the traditional approach welcomes the increased leverage $L=D / S$, and the associated increased of company capitalization (Brusov et al. 2013).The traditional (empirical) approach has existed up to appearance of the first quantitative theory by Modigliani and Miller in 1958 (Modigliani F., Miller M. 1958).

\section{MODIGLIANI-MILLER THEORY}

\subsection{Modigliani-Miller Theory without Taxes}

Modigliani and Miller (MM) in their first paper (Modigliani F., Miller M. 1958) come to conclusions which are fundamentally different from the conclusions of traditional approach. Under assumptions (see Appendix), that there is no taxes, no transaction costs, no bankruptcy costs, perfect market exists with symmetry information, equivalence in borrowing costs for both companies and investors etc. they showed that choosing of proportion of debt and equity does not affect company value as well as the weighted average cost of capital, WACC (Figure 1).

Most of Modigliani and Miller assumptions (Modigliani F., Miller M. 1958), of course, are unrealistic. Some assumptions can be removed without changing the conclusions of the model. However, assuming no costs of bankruptcy and the absence of taxes (or the presence of only corporate taxes) are crucial - the change of these assumptions alters conclusions. The last two assumptions rule out the possibility of signaling and agency costs and, thus, also constitute a critical prerequisite.
The most crucial changes of Modigliani - Miller theory, however, turn out to be due to the refusal of perpetuity of the companies. These changes have led to creation by Brusov-Filatova-Orekhova (Brusov et al. 2013, 2011a, 2011b, 2011c, 2012a, 2012b) a modern theory of capital cost and capital structure (BFO theory), which has replaced the Modigliani - Miller theory. Within BFO theory a few very important effects in corporate finance have been discovered, which are absent in the Modigliani - Miller theory (see Paragraph 4).

\subsection{Modigliani-Miller Theory with Taxes}

In the real situation taxes on profits of companies always exist. Since the interest paid on debt, are excluded from the tax base - it leads to the so- called effect of "tax shield": value of the company, used the borrowed capital (leverage company), is higher than the value of the company, financed entirely by the equity (non- leverage company). The value of the "tax shield" for one year is equal to $k_{d} D t$, where $D$ is the value of debt, $t$ - the income tax rate, $k_{d}$ - the interest on the debt (Modigliani F., Miller M. 1963). The value of the "tax shield" for perpetuity company for all time of its existence is equal to (we used the formula for the sum of terms of an infinitely decreasing geometric progression),

$$
(P V)_{T S}=k_{d} D t \sum_{t=1}^{\infty}\left(1+k_{d}\right)^{-t}=D t
$$

and the cost of leverage company is equal to

$$
V=V_{0}+D t
$$

where $V_{0}$ is the value of financially independent company.

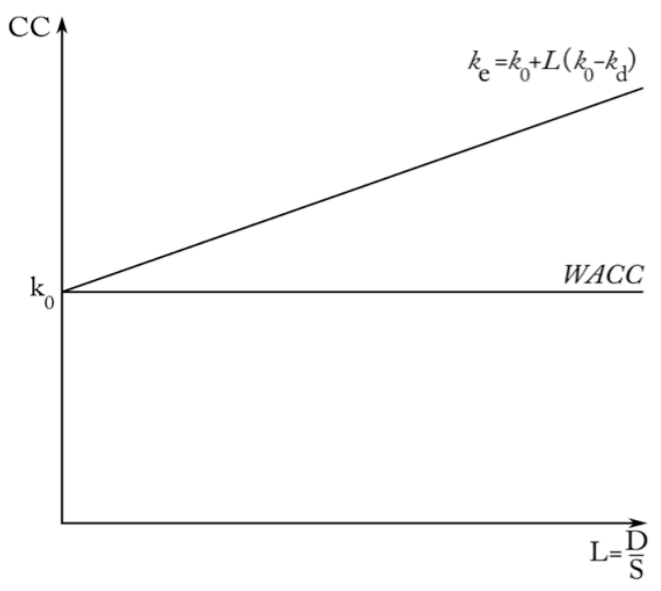

Figure 1: Modigliani-Miller theory without taxes. 
Thus, we obtain the following result obtained by Modigliani and Miller in 1963 (Modigliani F., Miller M. 1963)

The value of financially dependent company is equal to the value of the company of the same risk group used no leverage, increased by the value of tax shield arising from financial leverage and equal to the product of rate of corporate income tax $T$ and the value of debt $D$.

The following expression has been derived by Modigliani and Miller for the cost of equity capital

$k_{e}=k_{0}+L\left(k_{0}-k_{d}\right)(1-T)$

Thus, we can formulate the following conclusion by Modigliani and Miller.

The cost of equity of finance dependent company, which pays taxes on profits, is equal to the cost of equity of finance independent company of the same risk, increased by the magnitude of the risk premium, size of which depends on the difference between the cost of equity of finance independent company and borrowed capital, and on the value of used debt as well as on the tax rate on company profits.

Within their theory Modigliani and Miller had come to the following conclusions. With the growth of financial leverage (Figure 2):

1) the company value increases,

2) the weighted average cost of capital decreases from $k_{0}$ (for $\left.L=0\right)$ up to $k_{0}(1-T)$ (for $L=\infty$, when the company is financed entirely with borrowed funds).

3) the cost of equity capital increases linearly from $k_{0}$ (for $L=0$ ) up to $\infty$ (for $L=\infty$ ).

\section{TRADEOFF THEORY}

Reduction in financial sustainability companies and increase of bankruptcy risk, which relate to the use of different forms of borrowing in the formation of financial capital structure of the company, with the increasing of debt is increased.

Modigliani-Miller theory did not take into account the bankruptcy risk and related costs. From its version with the profit tax it follows that, debt financing brings only some benefits associated with tax benefits (tax shield). Because company capitalization grows with leverage and there is no compensating increase in the debt cost, increasing the capitalization requires use of debt financing only.

This obvious contradiction with the real economy has created many theories, which had tried to find a balance between the advantages and disadvantages of using by the companies of debt financing. The advantage is a reduction of weighted average cost of capital, WACC, and the corresponding increase of capitalization of the companies, V, and the drawback reduce with the increase of debt financing of financial sustainability of the companies and increased financial distress costs and risk of bankruptcy.

One of these theories is tradeoff theory (Brennan M., Schwartz E. 1978, Leland H. 1994). There are two

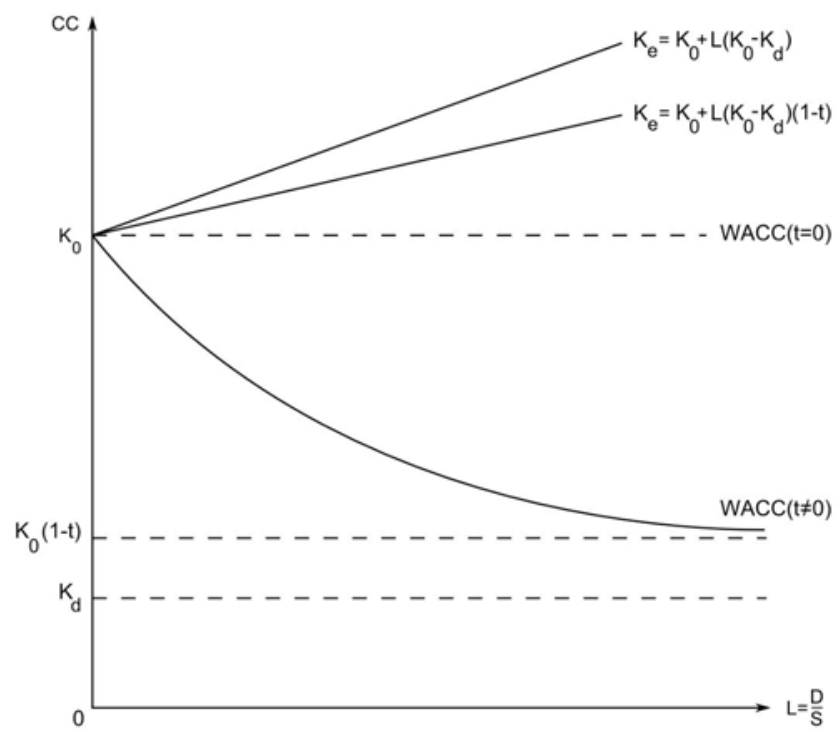

Figure 2: Dependence of equity capital cost, debt cost and WACC on leverage without taxes $(t=0)$ and with taxes $(t \neq 0)$. 
version of this theory: static and dynamic. Former one is based on the fact that at the low leverage level the benefits of debt financing are manifested: WACC drops with leverage, a company capitalization is growing.

Starting with a certain leverage level financial distress costs and risk of bankruptcy are growing, the WACC begins to growth and the value of the company begins to fall. The leverage level, at which the value of tax benefits is approximately equal to the cost of bankruptcy, determines the optimal (objective) capital structure.

While the static trade-off theory is single-period model (Brennan M., Schwartz E. 1978, Leland H. 1994), in the dynamic trade-off theory (Brennan, M. J., and E. S. Schwartz. 1984, Kane, A., A. J. Marcus, and R. L. McDonald. 1984) the financing decision depends on what the company anticipates in the next periods, which will be a capital structure.

\section{MODERN THEORY OF CAPITAL COST AND CAPITAL STRUCTURE BY BRUSOV-FILATOVA- OREKHOVA (BFO THEORY)}

Modigliani and Miller (Modigliani F., Miller M. 1958, 1963, 1966) assumed that all financial flows are perpetuity. Because, in reality the life time of the companies are always, of course, finite, this condition is one of the weaknesses of the Modigliani and Miller theory. Account of the finite life time of the companies changes all the formulas of Modigliani and Miller drastically. The solution of the problem of weighted average cost of capital, WACC, for the companies with arbitrary life time has been done for the first time by Brusov-Filatova-Orekhova with coauthors (Brusov et al. 2013, 2011a, 2011b, 2011c, 2012a, 2012b). Their theory has allowed to find hidden global causes of the global financial crisis (Brusov et al. 2012b).

The main formula, received by them, is an algebraic equation of $n+1$ power ( $n$ is a term of life of company) to calculate weighted average cost of capital, WACC, taking the form

$$
\frac{\left[1-(1+W A C C)^{-n}\right]}{W A C C}=\frac{\left[1-\left(1+k_{0}\right)^{-n}\right]}{k_{0}\left[1-\omega_{d} T\left(1-\left(1+k_{d}\right)^{-n}\right)\right]} .
$$

For $\mathrm{n}>3$ this equation can be solved numerically only. It is easy to use for this a function "matching parameters" in the Excel.Using equation (4), let us investigate the optimal capital structure in the tradeoff theory.

\section{ANALYSIS OF THE TRADEOFF THEORY BY USING THE THEORY BY BRUSOV-FILATOVA- OREKHOVA}

We are modeling the emergence of a financial volatility and of bankruptcy risk by the growth of the cost of debt capital $k_{d}$, indicating that $k_{d}$ becomes risky and its growth represents a fee for the state of financial volatility and bankruptcy risk.

It is impossible to study such effects, as the growth of credit rate with leverage in the theory of Modigliani and Miller, because:

MM theory considers two types of assets: risky equity capital and free of risk debt capital;

weighted average cost of capital, WACC, in the theory of Modigliani and Miller is determined by the following expression

$W A C C=k_{0}\left(1-w_{d} T\right)$,

which depends on $k_{0}, w_{d}, T$ and does NOT depends on $k_{d}$.

This is due to the fact that discounted value of tax shields for an infinite period of time

$(P V)_{T S}=k_{d} D T \sum_{t=1}^{\infty}\left(1+k_{d}\right)^{-t}=D T$

with the use of $k_{d}$ as discount rate does NOT depend on $k_{d}$.

In contrast to the theory of the Modigliani and Miller, in a modern theory of capital cost and capital structure of the company by Brusov-Filatova-Orekhova (BFO theory) (Brusov et al. 2013, 2011a, 2011b, 2011c, 2012a, 2012b) (Brusov et al. 2013, 2011a, 2011b, 2011c, 2012a, 2012b) discounted value of tax shields is valuated for finite period of time $n$ (term of life of company or the time from the establishment of companies up to the present moment $(n))$ and depends on $k_{d}$

$$
(P V)_{T S}=k_{d} D T \sum_{t=1}^{n}\left(1+k_{d}\right)^{-t}=D T\left[1-\left(1+k_{d}\right)^{-n}\right],
$$

as well as capitalization of financially independent

$$
V_{0}=C F\left[1-\left(1+k_{0}\right)^{-n}\right] / k_{0}
$$

and capitalization of financially dependent company 
$V=C F\left[1-(1+W A C C)^{-n}\right] / W A C C$.

As a result, for weighted average cost of capital WACC the formula BFO is derived

$$
\frac{\left[1-(1+W A C C)^{-n}\right]}{W A C C}=\frac{\left[1-\left(1+k_{0}\right)^{-n}\right]}{k_{0}\left[1-\omega_{d} T\left(1-\left(1+k_{d}\right)^{-n}\right)\right]}
$$

1. $n=3 ; t=20 \% ; L=0,1,2, \ldots 10$

$k_{0}=24 \% ; \bar{k}_{d}=\left\{\begin{array}{c}0,07 ; a t L \leq 2 \\ 0,07+0,01(L-2)^{2} ; \text { at } L>2\end{array}\right\}$

Table 1:

\begin{tabular}{|c|c||c|c|c|c|c|c|c|c|c|c|c|}
\hline $\mathbf{n}$ & $\mathbf{L}$ & $\mathbf{0}$ & $\mathbf{1}$ & $\mathbf{2}$ & $\mathbf{3}$ & $\mathbf{4}$ & $\mathbf{5}$ & $\mathbf{6}$ & $\mathbf{7}$ & $\mathbf{8}$ & $\mathbf{9}$ & $\mathbf{1 0}$ \\
\hline \hline $\mathbf{3}$ & $\mathbf{k d}$ & 0,07 & 0,07 & 0,07 & 0,08 & 0,11 & 0,16 & 0,23 & 0,32 & 0,43 & 0,56 & 0,71 \\
\hline $\mathbf{k 0}$ & $\mathbf{A}$ & 1,9813 & 2,0184 & 2,0311 & 2,0445 & 2,0703 & 2,1075 & 2,1520 & 2,1988 & 2,2438 & 2,2842 & 2,3186 \\
\hline $\mathbf{0 , 2 4}$ & WACC & 0,2401 & 0,2279 & 0,2238 & 0,2195 & 0,2111 & 0,1997 & 0,1864 & 0,1730 & 0,1605 & 0,1496 & 0,1406 \\
\hline
\end{tabular}

\section{WACC(L)}

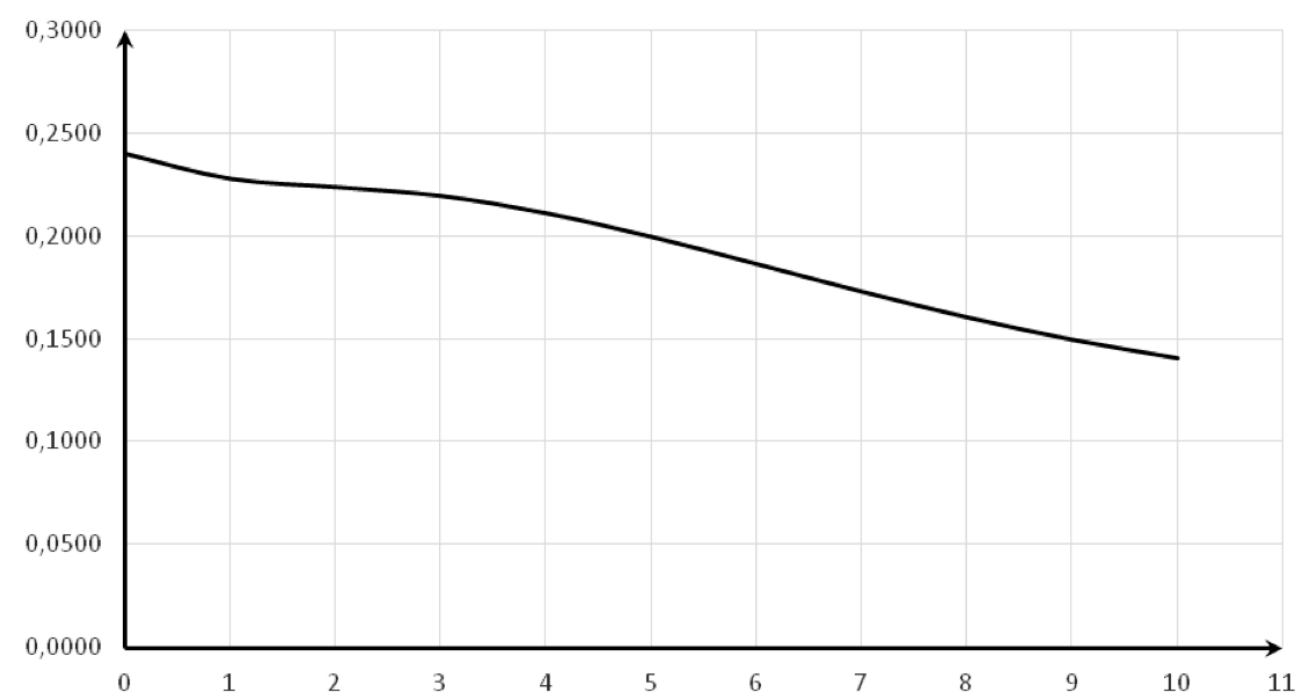

Figure 3: Dependence of WACC on L.

2. $n=5 ; t=20 \% ; L=0,1,2, \ldots 10$

$k_{0}=24 \% ; \bar{k}_{d}=\left\{\begin{array}{c}0,07 ; a t L \leq 2 \\ 0,07+0,01(L-2)^{2} ; \text { at } L>2\end{array}\right\}$

Table 2:

\begin{tabular}{|c|c||c|c|c|c|c|c|c|c|c|c|c|}
\hline $\mathbf{n}$ & $\mathbf{L}$ & $\mathbf{0}$ & $\mathbf{1}$ & $\mathbf{2}$ & $\mathbf{3}$ & $\mathbf{4}$ & $\mathbf{5}$ & $\mathbf{6}$ & $\mathbf{7}$ & $\mathbf{8}$ & $\mathbf{9}$ & $\mathbf{1 0}$ \\
\hline \hline $\mathbf{5}$ & $\mathbf{k d}$ & 0,07 & 0,07 & 0,07 & 0,08 & 0,11 & 0,16 & 0,23 & 0,32 & 0,43 & 0,56 & 0,71 \\
\hline $\mathbf{k 0}$ & $\mathbf{A}$ & 2,7454 & 2,8265 & 2,8546 & 2,8835 & 2,9364 & 3,0080 & 3,0866 & 3,1605 & 3,2225 & 3,2703 & 3,3052 \\
\hline $\mathbf{0 , 2 4}$ & WACC & 0,2400 & 0,2261 & 0,2215 & 0,2168 & 0,2083 & 0,1973 & 0,1858 & 0,1753 & 0,1669 & 0,1605 & 0,1560 \\
\hline
\end{tabular}




\section{WACC(L)}

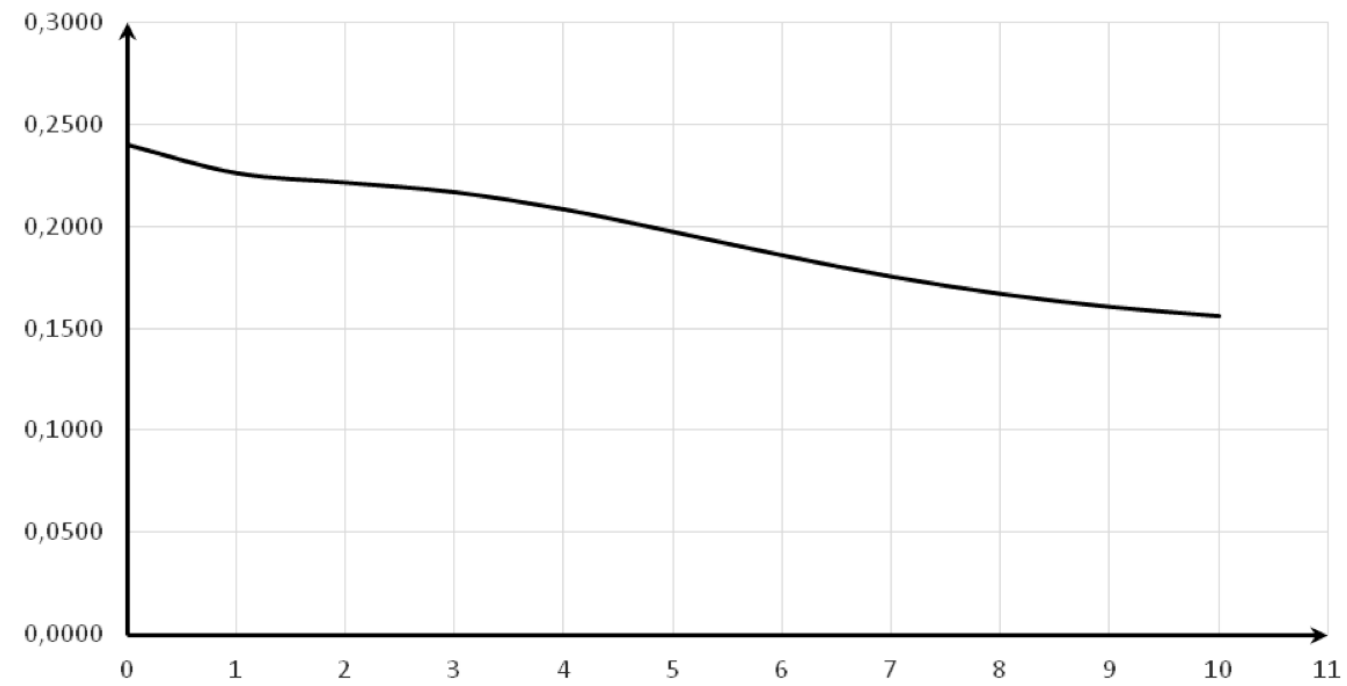

Figure 4: Dependence of WACC on L.

3. $n=3 ; t=20 \% ; L=0,1,2, \ldots 10$

$k_{0}=24 \% ; \bar{k}_{d}=\left\{\begin{array}{c}0,07 ; \text { at } L \leq 2 \\ 0,07+0,1(L-2)^{2} ; \text { at } L>2\end{array}\right\}$

Table 3:

\begin{tabular}{|c|c||c|c|c|c|c|c|c|c|c|c|c|}
\hline $\mathbf{n}$ & $\mathbf{L}$ & $\mathbf{0}$ & $\mathbf{1}$ & $\mathbf{2}$ & $\mathbf{3}$ & $\mathbf{4}$ & $\mathbf{5}$ & $\mathbf{6}$ & $\mathbf{7}$ & $\mathbf{8}$ & $\mathbf{9}$ & $\mathbf{1 0}$ \\
\hline \hline $\mathbf{3}$ & $\mathbf{k d}$ & 0,07 & 0,07 & 0,07 & 0,17 & 0,47 & 0,97 & 1,67 & 2,57 & 3,67 & 4,97 & 6,47 \\
\hline k0 & $\mathbf{A}$ & 1,9813 & 2,0184 & 2,0311 & 2,0996 & 2,2253 & 2,3170 & 2,3655 & 2,3904 & 2,4046 & 2,4137 & 2,4203 \\
\hline $\mathbf{0 , 2 4}$ & WACC & 0,2401 & 0,2279 & 0,2238 & 0,2021 & 0,1656 & 0,1410 & 0,1289 & 0,1228 & 0,1193 & 0,1171 & 0,1156 \\
\hline
\end{tabular}

\section{WACC(L)}

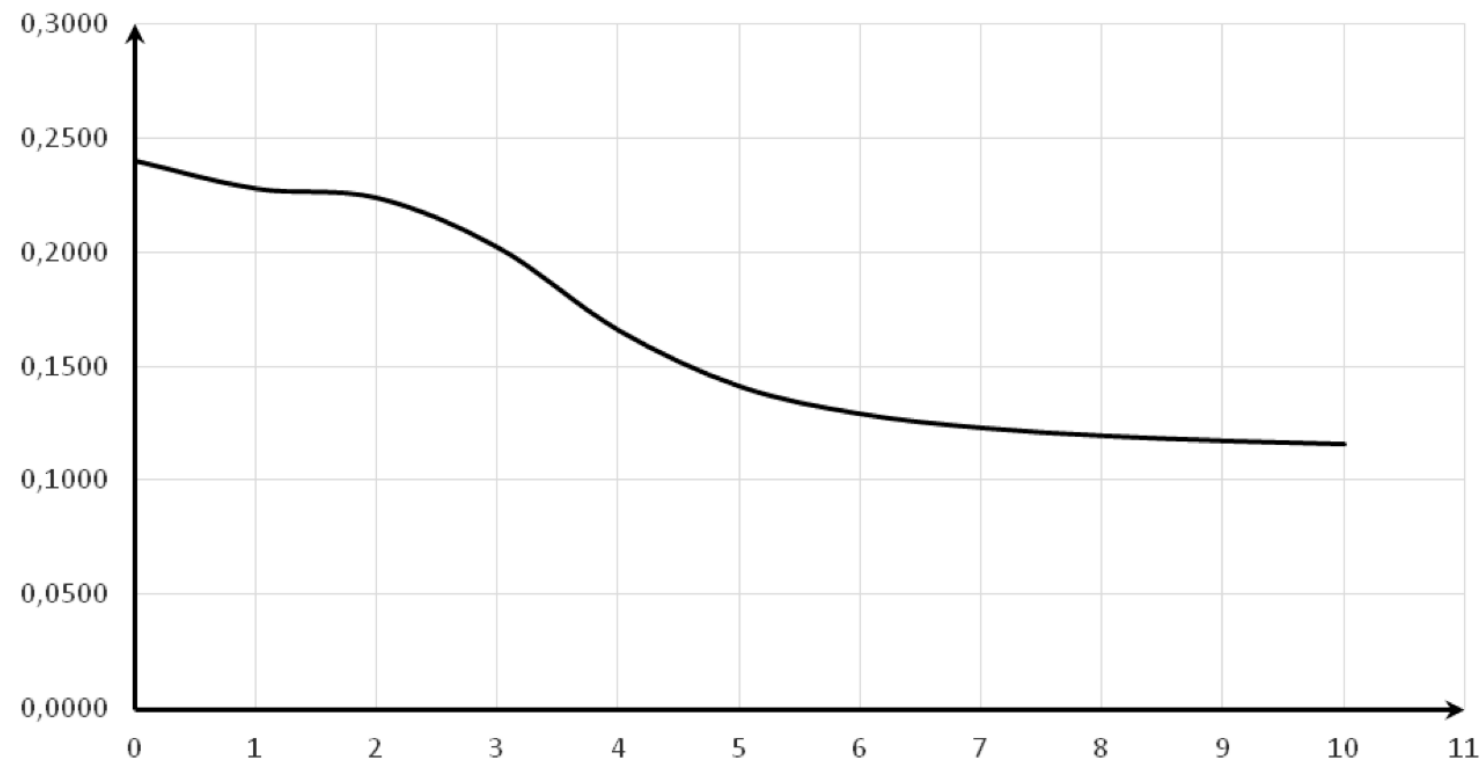

Figure 5: Dependence of WACC on L. 
4. $n=5 ; t=20 \% ; L=0,1,2, \ldots 10$

$k_{0}=24 \% ; \bar{k}_{d}=\left\{\begin{array}{c}0,07 ; \text { at } L \leq 2 \\ 0,07+0,1(L-2)^{2} ; \text { at } L>2\end{array}\right\}$

Table 4:

\begin{tabular}{|c|c||c|c|c|c|c|c|c|c|c|c|c|}
\hline $\mathbf{n}$ & $\mathbf{L}$ & $\mathbf{0}$ & $\mathbf{1}$ & $\mathbf{2}$ & $\mathbf{3}$ & $\mathbf{4}$ & $\mathbf{5}$ & $\mathbf{6}$ & $\mathbf{7}$ & $\mathbf{8}$ & $\mathbf{9}$ & $\mathbf{1 0}$ \\
\hline \hline $\mathbf{5}$ & $\mathbf{k d}$ & 0,07 & 0,07 & 0,07 & 0,17 & 0,47 & 0,97 & 1,67 & 2,57 & 3,67 & 4,97 & 6,47 \\
\hline $\mathbf{k 0}$ & $\mathbf{A}$ & 2,7454 & 2,8265 & 2,8546 & 2,9893 & 3,1801 & 3,2724 & 3,3084 & 3,3265 & 3,3387 & 3,3479 & 3,3554 \\
\hline $\mathbf{0 , 2 4}$ & WACC & 0,2400 & 0,2261 & 0,2215 & 0,2001 & 0,1726 & 0,1603 & 0,1556 & 0,1533 & 0,1517 & 0,1506 & 0,1496 \\
\hline
\end{tabular}

\section{WACC(L)}

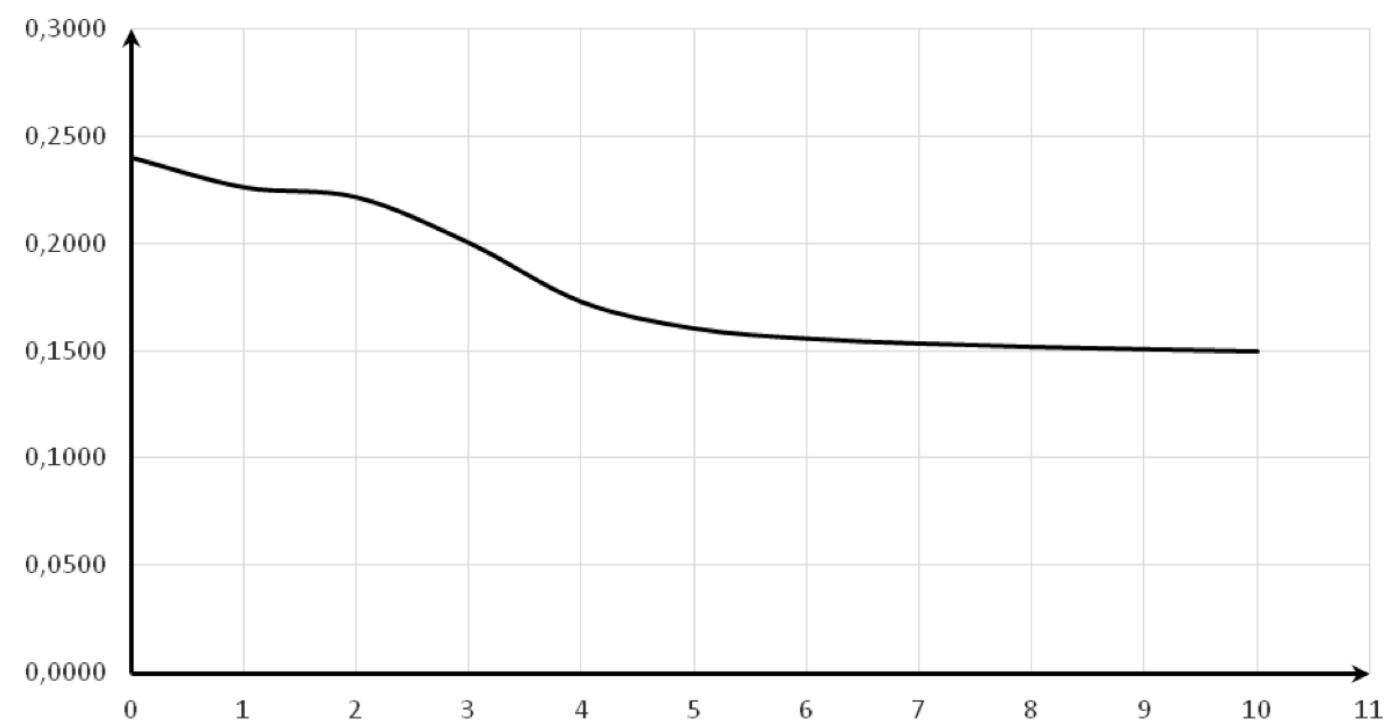

Figure 6: Dependence of WACC on L.

5. $n=3 ; t=20 \% ; L=0,1,2, \ldots 10$

$k_{0}=24 \% ; \bar{k}_{d}=\left\{\begin{array}{c}0,07 ; \text { at } L \leq 2 \\ 0,07+0,01(L-2) ; \text { at } L>2\end{array}\right\}$

Table 5:

\begin{tabular}{|c|c||c|c|c|c|c|c|c|c|c|c|c|}
\hline $\mathbf{n}$ & $\mathbf{L}$ & $\mathbf{0}$ & $\mathbf{1}$ & $\mathbf{2}$ & $\mathbf{3}$ & $\mathbf{4}$ & $\mathbf{5}$ & $\mathbf{6}$ & $\mathbf{7}$ & $\mathbf{8}$ & $\mathbf{9}$ & $\mathbf{1 0}$ \\
\hline \hline $\mathbf{3}$ & $\mathbf{k d}$ & 0,07 & 0,07 & 0,07 & 0,08 & 0,09 & 0,1 & 0,11 & 0,12 & 0,13 & 0,14 & 0,15 \\
\hline $\mathbf{k 0}$ & $\mathbf{A}$ & 1,9813 & 2,0184 & 2,0311 & 2,0445 & 2,0563 & 2,0670 & 2,0770 & 2,0865 & 2,0957 & 2,1044 & 2,1129 \\
\hline $\mathbf{0 , 2 4}$ & WACC & 0,2401 & 0,2279 & 0,2238 & 0,2195 & 0,2159 & 0,2122 & 0,2090 & 0,2061 & 0,2033 & 0,2006 & 0,1981 \\
\hline
\end{tabular}




\section{WACC(L)}

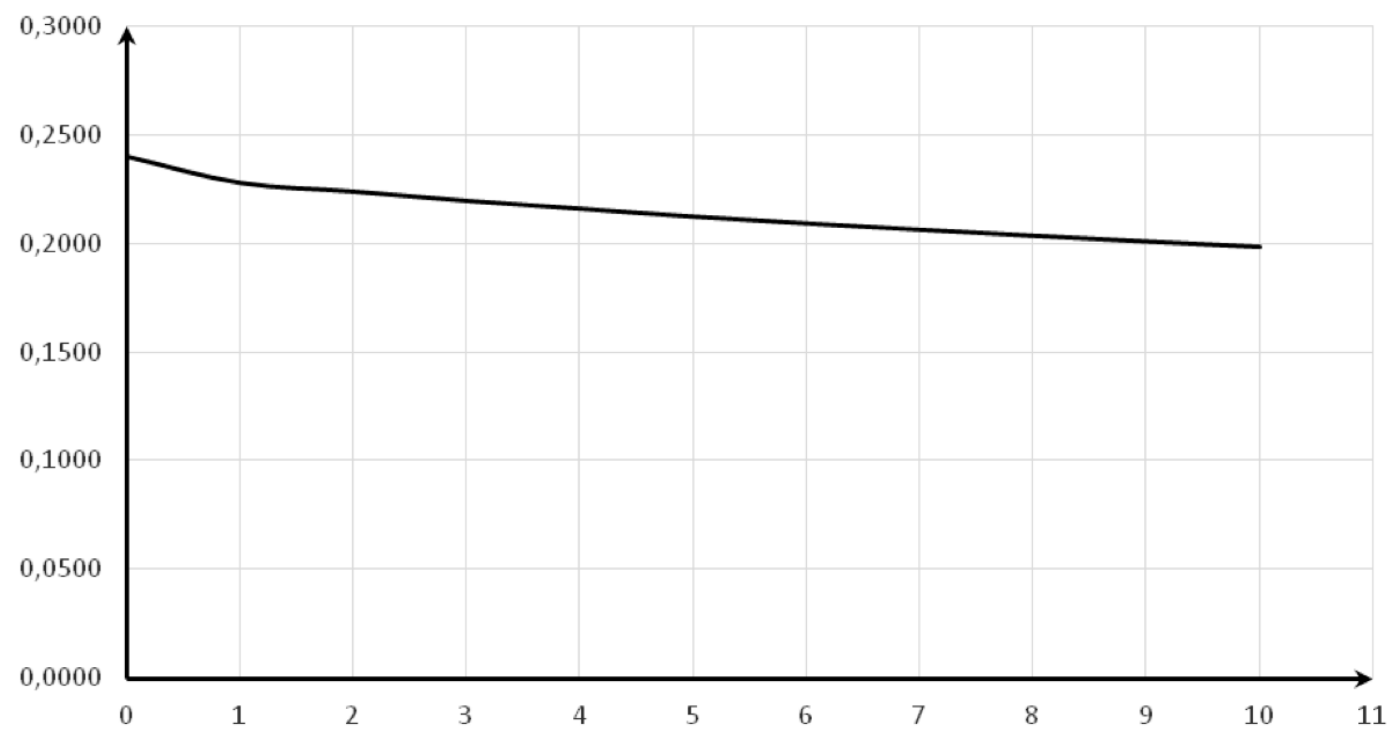

Figure 7: Dependence of WACC on L.

6. $n=5 ; t=20 \% ; L=0,1,2, \ldots 10$

$k_{0}=24 \% ; \bar{k}_{d}=\left\{\begin{array}{c}0,07 ; \text { at } L \leq 2 \\ 0,07+0,01(L-2) ; \text { at } L>2\end{array}\right\}$

Table 6:

\begin{tabular}{|c|c||c|c|c|c|c|c|c|c|c|c|c|}
\hline $\mathbf{n}$ & $\mathbf{L}$ & $\mathbf{0}$ & $\mathbf{1}$ & $\mathbf{2}$ & $\mathbf{3}$ & $\mathbf{4}$ & $\mathbf{5}$ & $\mathbf{6}$ & $\mathbf{7}$ & $\mathbf{8}$ & $\mathbf{9}$ & $\mathbf{1 0}$ \\
\hline \hline $\mathbf{5}$ & $\mathbf{k d}$ & 0,07 & 0,07 & 0,07 & 0,08 & 0,09 & 0,1 & 0,11 & 0,12 & 0,13 & 0,14 & 0,15 \\
\hline $\mathbf{k 0}$ & $\mathbf{A}$ & 2,7454 & 2,8265 & 2,8546 & 2,8835 & 2,9083 & 2,9305 & 2,9511 & 2,9702 & 2,9883 & 3,0054 & 3,0216 \\
\hline $\mathbf{0 , 2 4}$ & WACC & 0,2400 & 0,2261 & 0,2215 & 0,2168 & 0,2128 & 0,2093 & 0,2060 & 0,2031 & 0,2003 & 0,1977 & 0,1952 \\
\hline
\end{tabular}

WACC(L)

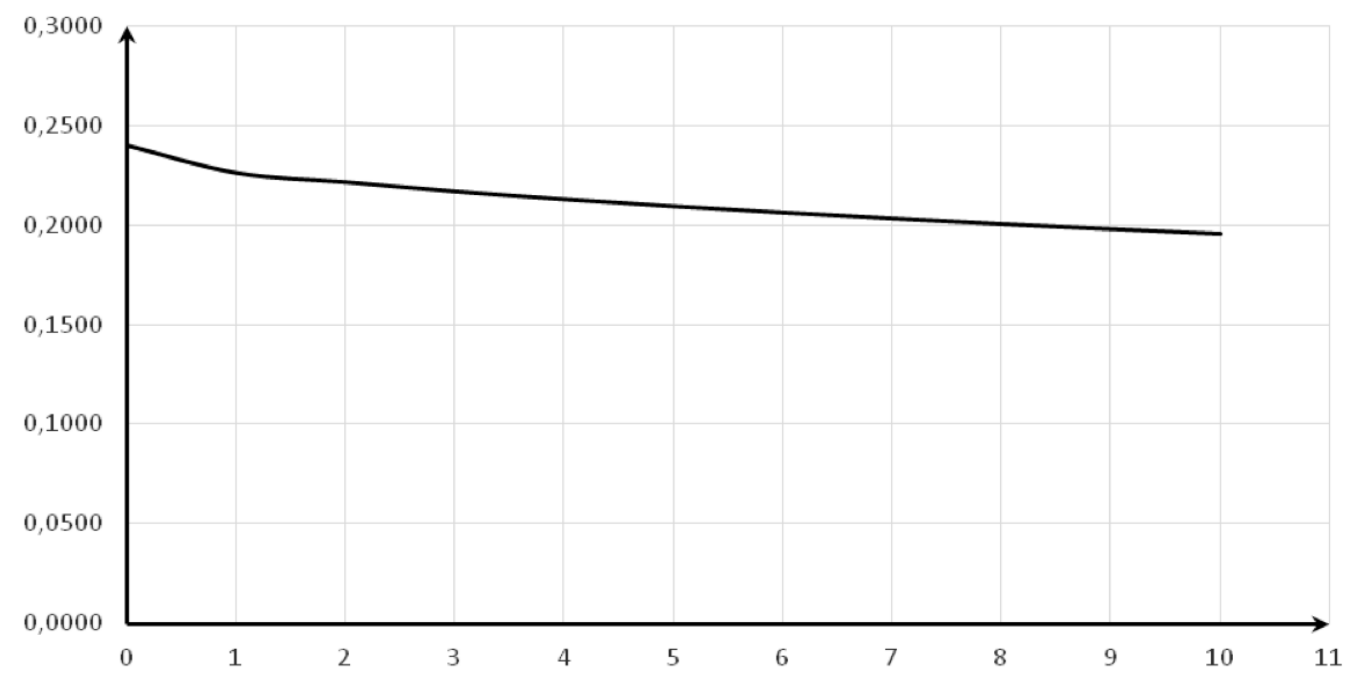

Figure 8: Dependence of WACC on L. 
7. $n=3 ; t=20 \% ; L=0,1,2, \ldots 10$

$k_{0}=24 \% ; \bar{k}_{d}=\left\{\begin{array}{c}0,07 ; \text { at } L \leq 2 \\ 0,07+0,1(L-2) ; \text { at } L>2\end{array}\right\}$

Table 7:

\begin{tabular}{|c|c||c|c|c|c|c|c|c|c|c|c|c|}
\hline $\mathbf{n}$ & $\mathbf{L}$ & $\mathbf{0}$ & $\mathbf{1}$ & $\mathbf{2}$ & $\mathbf{3}$ & $\mathbf{4}$ & $\mathbf{5}$ & $\mathbf{6}$ & $\mathbf{7}$ & $\mathbf{8}$ & $\mathbf{9}$ & $\mathbf{1 0}$ \\
\hline \hline $\mathbf{3}$ & $\mathbf{k d}$ & 0,07 & 0,07 & 0,07 & 0,17 & 0,27 & 0,37 & 0,47 & 0,57 & 0,67 & 0,77 & 0,87 \\
\hline $\mathbf{k 0}$ & $\mathbf{A}$ & 1,9813 & 2,0184 & 2,0311 & 2,0996 & 2,1580 & 2,2060 & 2,2450 & 2,2768 & 2,3028 & 2,3242 & 2,3420 \\
\hline $\mathbf{0 , 2 4}$ & WACC & 0,2401 & 0,2279 & 0,2238 & 0,2021 & 0,1847 & 0,1710 & 0,1602 & 0,1516 & 0,1447 & 0,1391 & 0,1346 \\
\hline
\end{tabular}

\section{WACC(L)}

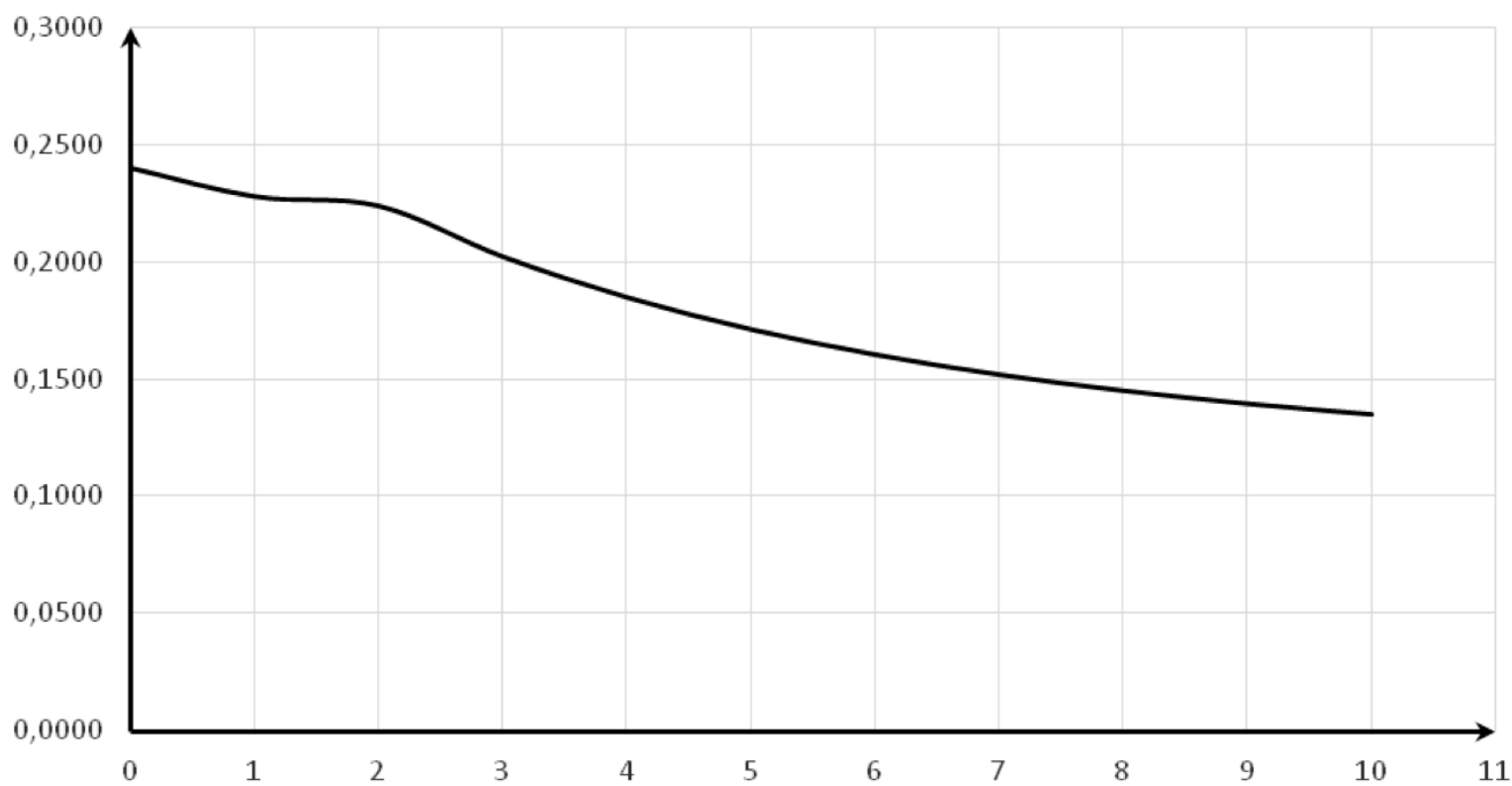

Figure 9: Dependence of WACC on L.

8. $n=5 ; t=20 \% ; L=0,1,2, \ldots 10$

$k_{0}=24 \% ; \bar{k}_{d}=\left\{\begin{array}{c}0,07 ; \text { at } L \leq 2 \\ 0,07+0,1(L-2) ; \text { at } L>2\end{array}\right\}$

Table 8:

\begin{tabular}{|c|c||c|c|c|c|c|c|c|c|c|c|c|}
\hline $\mathbf{n}$ & $\mathbf{L}$ & $\mathbf{0}$ & $\mathbf{1}$ & $\mathbf{2}$ & $\mathbf{3}$ & $\mathbf{4}$ & $\mathbf{5}$ & $\mathbf{6}$ & $\mathbf{7}$ & $\mathbf{8}$ & $\mathbf{9}$ & $\mathbf{1 0}$ \\
\hline \hline $\mathbf{5}$ & $\mathbf{k d}$ & 0,07 & 0,07 & 0,07 & 0,17 & 0,27 & 0,37 & 0,47 & 0,57 & 0,67 & 0,77 & 0,87 \\
\hline $\mathbf{k 0}$ & $\mathbf{A}$ & 2,7454 & 2,8265 & 2,8546 & 2,9893 & 3,0902 & 3,1634 & 3,2164 & 3,2553 & 3,2843 & 3,3063 & 3,3232 \\
\hline $\mathbf{0 , 2 4}$ & WACC & 0,2400 & 0,2261 & 0,2215 & 0,2001 & 0,1853 & 0,1749 & 0,1677 & 0,1625 & 0,1587 & 0,1559 & 0,1537 \\
\hline
\end{tabular}




\section{WACC(L)}

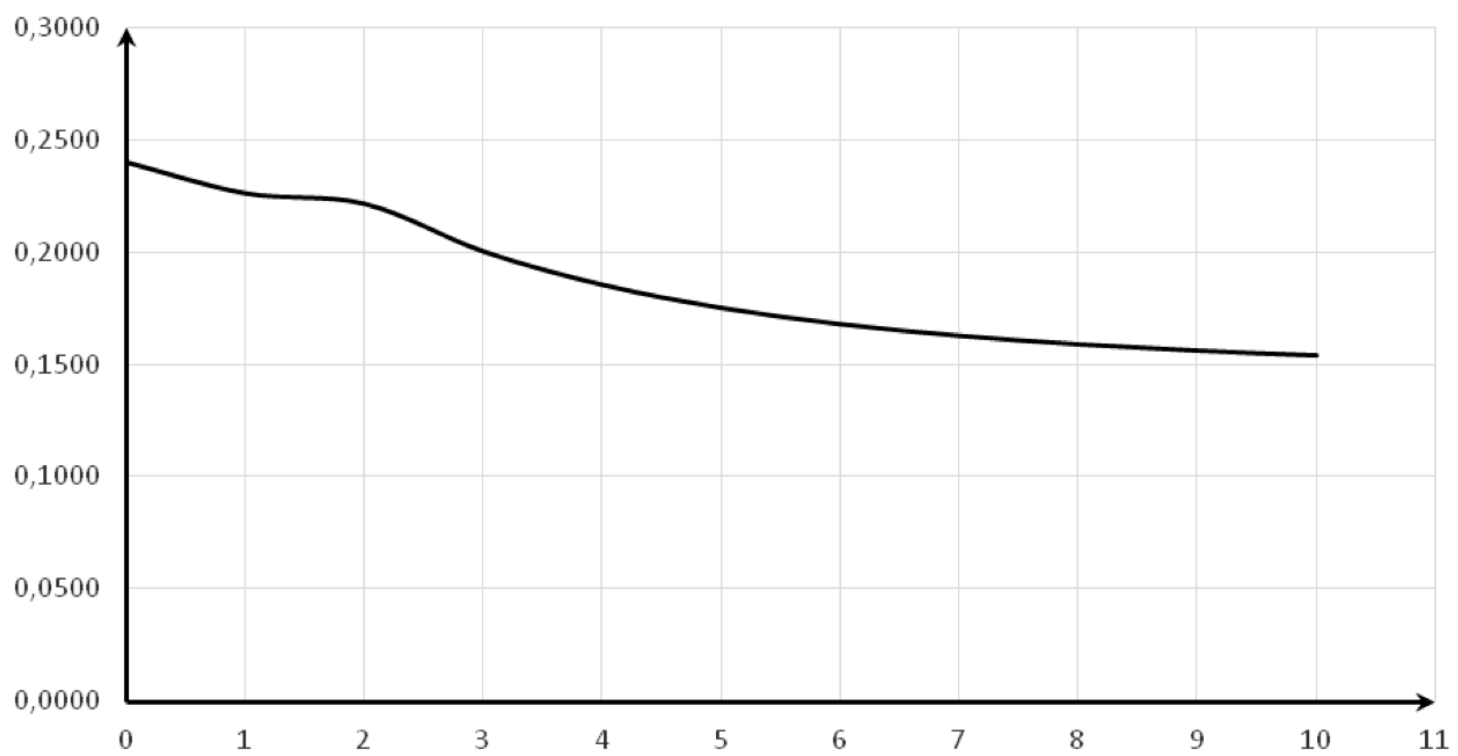

Figure 10: Dependence of WACC on L.

9. $n=3 ; t=20 \% ; L=0,1,2, \ldots 10$

$k_{0}=12 \% ; \bar{k}_{d}=\left\{\begin{array}{c}0,07 ; a t L \leq 2 \\ 0,07+0,01(L-2)^{2} ; \text { at } L>2\end{array}\right\}$

Table 9:

\begin{tabular}{|c|c|c|c|c|c|c|c|c|c|c|c|c|}
\hline $\mathbf{n}$ & $\mathbf{L}$ & $\mathbf{0}$ & $\mathbf{1}$ & $\mathbf{2}$ & $\mathbf{3}$ & $\mathbf{4}$ & $\mathbf{5}$ & $\mathbf{6}$ & $\mathbf{7}$ & $\mathbf{8}$ & $\mathbf{9}$ & $\mathbf{1 0}$ \\
\hline \hline $\mathbf{3}$ & $\mathbf{k d}$ & 0,07 & 0,07 & 0,07 & 0,08 & 0,11 & 0,16 & 0,23 & 0,32 & 0,43 & 0,56 & 0,71 \\
\hline $\mathbf{k 0}$ & $\mathbf{A}$ & 2,4018 & 2,4468 & 2,4621 & 2,4785 & 2,5098 & 2,5548 & 2,6087 & 2,6655 & 2,7200 & 2,7690 & 2,8107 \\
\hline $\mathbf{0 , 1 2}$ & WACC & 0,1200 & 0,1093 & 0,1057 & 0,1019 & 0,0948 & 0,0849 & 0,0734 & 0,0615 & 0,0506 & 0,0412 & 0,0333 \\
\hline
\end{tabular}

\section{WACC(L)}

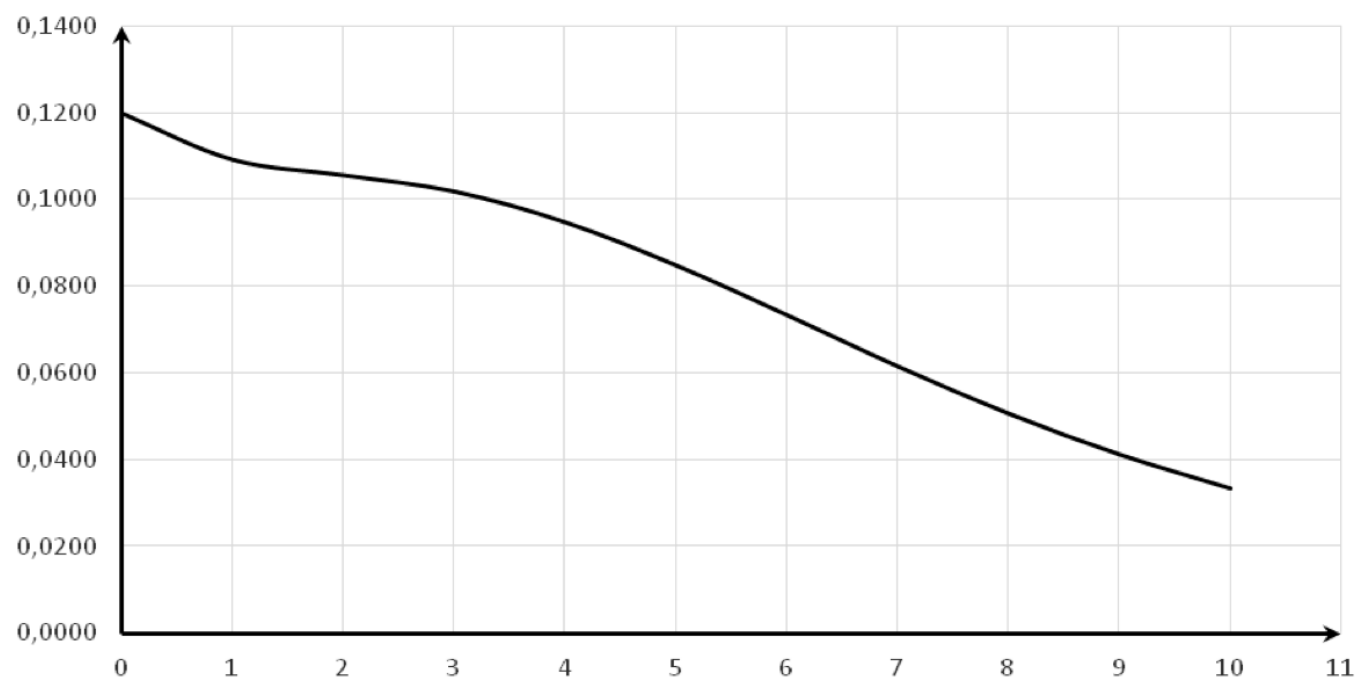

Figure 11: Dependence of WACC on L. 
10. $n=5 ; t=20 \% ; L=0,1,2, \ldots 10$

$k_{0}=12 \% ; \bar{k}_{d}=\left\{\begin{array}{c}0,07 ; \text { at } L \leq 2 \\ 0,07+0,01(L-2)^{2} ; \text { at } L>2\end{array}\right\}$

Table 10:

\begin{tabular}{|c|c||c|c|c|c|c|c|c|c|c|c|c|}
\hline $\mathbf{n}$ & $\mathbf{L}$ & $\mathbf{0}$ & $\mathbf{1}$ & $\mathbf{2}$ & $\mathbf{3}$ & $\mathbf{4}$ & $\mathbf{5}$ & $\mathbf{6}$ & $\mathbf{7}$ & $\mathbf{8}$ & $\mathbf{9}$ & $\mathbf{1 0}$ \\
\hline \hline $\mathbf{5}$ & $\mathbf{k d}$ & 0,07 & 0,07 & 0,07 & 0,08 & 0,11 & 0,16 & 0,23 & 0,32 & 0,43 & 0,56 & 0,71 \\
\hline $\mathbf{k 0}$ & $\mathbf{A}$ & 3,6048 & 3,7113 & 3,7482 & 3,7862 & 3,8556 & 3,9496 & 4,0528 & 4,1498 & 4,2312 & 4,2940 & 4,3399 \\
\hline $\mathbf{0 , 1 2}$ & WACC & 0,1200 & 0,1084 & 0,1045 & 0,1005 & 0,0934 & 0,0841 & 0,0744 & 0,0655 & 0,0584 & 0,0530 & 0,0492 \\
\hline
\end{tabular}

\section{WACC(L)}

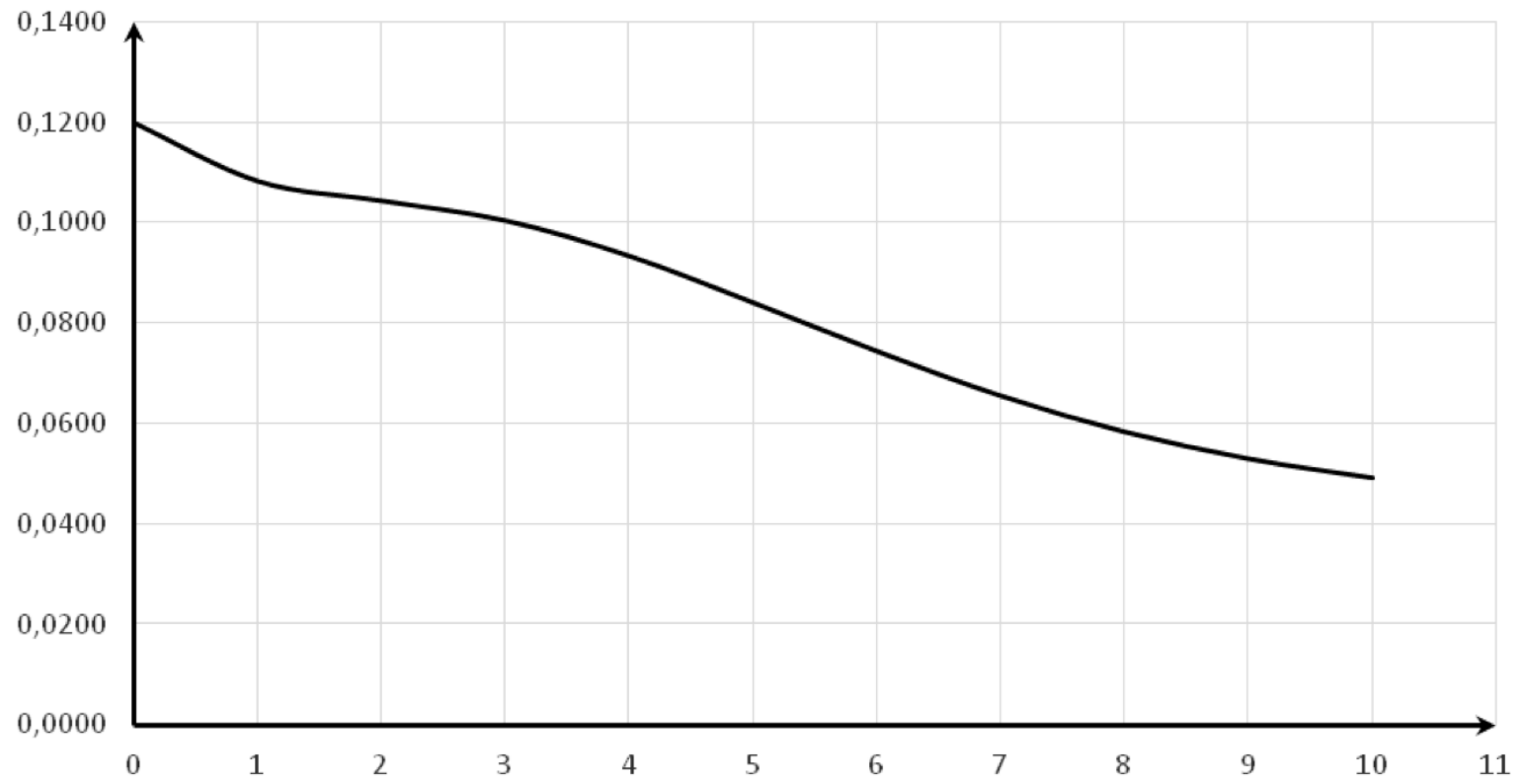

Figure 12: Dependence of WACC on L.

11. $n=3 ; t=20 \% ; L=0,1,2, \ldots 10$

$k_{0}=12 \% ; \bar{k}_{d}=\left\{\begin{array}{c}0,07 ; \text { at } L \leq 2 \\ 0,07+0,1(L-2)^{2} ; \text { at } L>2\end{array}\right\}$

\section{Table 11:}

\begin{tabular}{|c|c||c|c|c|c|c|c|c|c|c|c|c|}
\hline $\mathbf{n}$ & $\mathbf{L}$ & $\mathbf{0}$ & $\mathbf{1}$ & $\mathbf{2}$ & $\mathbf{3}$ & $\mathbf{4}$ & $\mathbf{5}$ & $\mathbf{6}$ & $\mathbf{7}$ & $\mathbf{8}$ & $\mathbf{9}$ & $\mathbf{1 0}$ \\
\hline \hline $\mathbf{3}$ & $\mathbf{k d}$ & 0,07 & 0,07 & 0,07 & 0,17 & 0,47 & 0,97 & 1,67 & 2,57 & 3,67 & 4,97 & 6,47 \\
\hline $\mathbf{k 0}$ & $\mathbf{A}$ & 2,4018 & 2,4468 & 2,4621 & 2,5452 & 2,6976 & 2,8087 & 2,8676 & 2,8978 & 2,9150 & 2,9260 & 2,9340 \\
\hline $\mathbf{0 , 1 2}$ & WACC & 0,1200 & 0,1093 & 0,1057 & 0,0870 & 0,0551 & 0,0337 & 0,0230 & 0,0176 & 0,0146 & 0,0127 & 0,0113 \\
\hline
\end{tabular}




\section{WACC(L)}

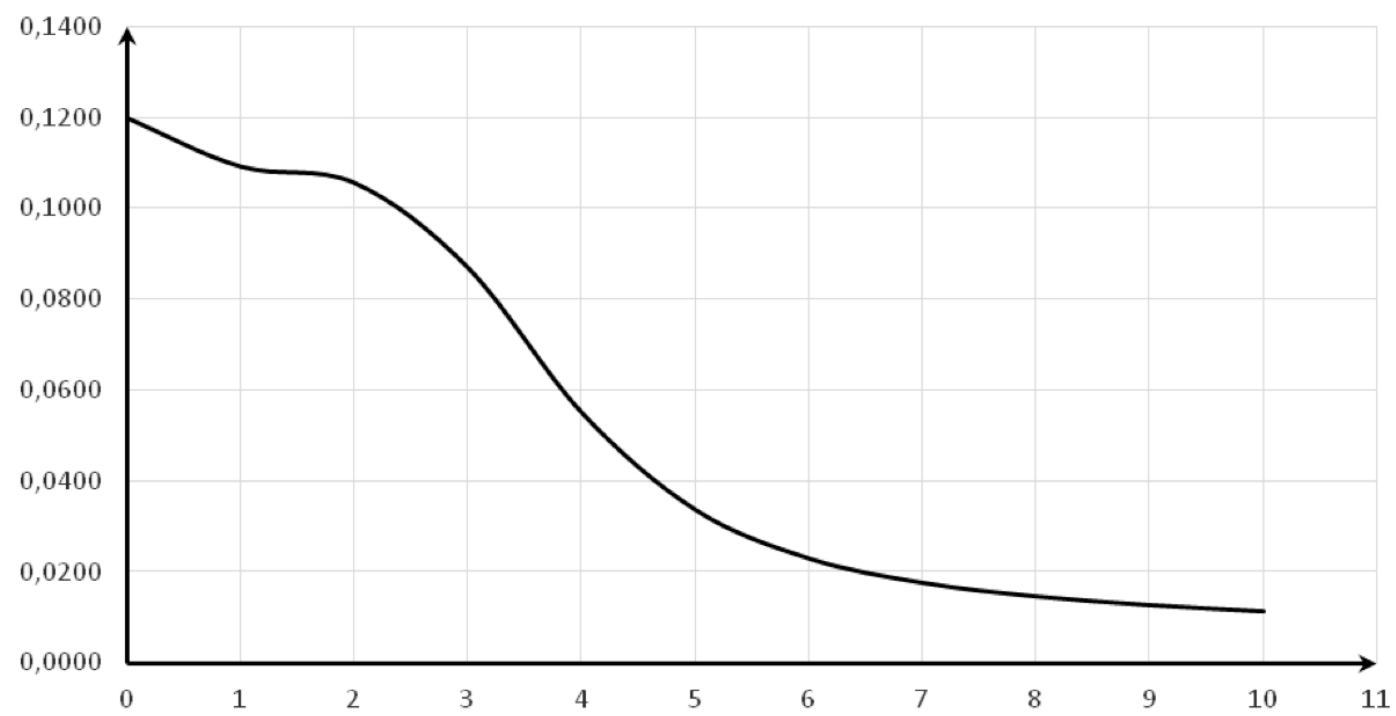

Figure 13: Dependence of WACC on L.

12. $n=5 ; t=20 \% ; L=0,1,2, \ldots 10$

$k_{0}=12 \% ; \bar{k}_{d}=\left\{\begin{array}{c}0,07 ; \text { at } L \leq 2 \\ 0,07+0,1(L-2)^{2} ; \text { at } L>2\end{array}\right\}$

Table 12:

\begin{tabular}{|c|c||c|c|c|c|c|c|c|c|c|c|c|}
\hline $\mathbf{n}$ & $\mathbf{L}$ & $\mathbf{0}$ & $\mathbf{1}$ & $\mathbf{2}$ & $\mathbf{3}$ & $\mathbf{4}$ & $\mathbf{5}$ & $\mathbf{6}$ & $\mathbf{7}$ & $\mathbf{8}$ & $\mathbf{9}$ & $\mathbf{1 0}$ \\
\hline \hline $\mathbf{5}$ & $\mathbf{k d}$ & 0,07 & 0,07 & 0,07 & 0,17 & 0,47 & 0,97 & 1,67 & 2,57 & 3,67 & 4,97 & 6,47 \\
\hline $\mathbf{k 0}$ & $\mathbf{A}$ & 3,6048 & 3,7113 & 3,7482 & 3,9250 & 4,1755 & 4,2968 & 4,3440 & 4,3678 & 4,3838 & 4,3959 & 4,4058 \\
\hline $\mathbf{0 , 1 2}$ & WACC & 0,1200 & 0,1084 & 0,1045 & 0,0866 & 0,0633 & 0,0528 & 0,0489 & 0,0468 & 0,0455 & 0,0445 & 0,0437 \\
\hline
\end{tabular}

\section{WACC(L)}

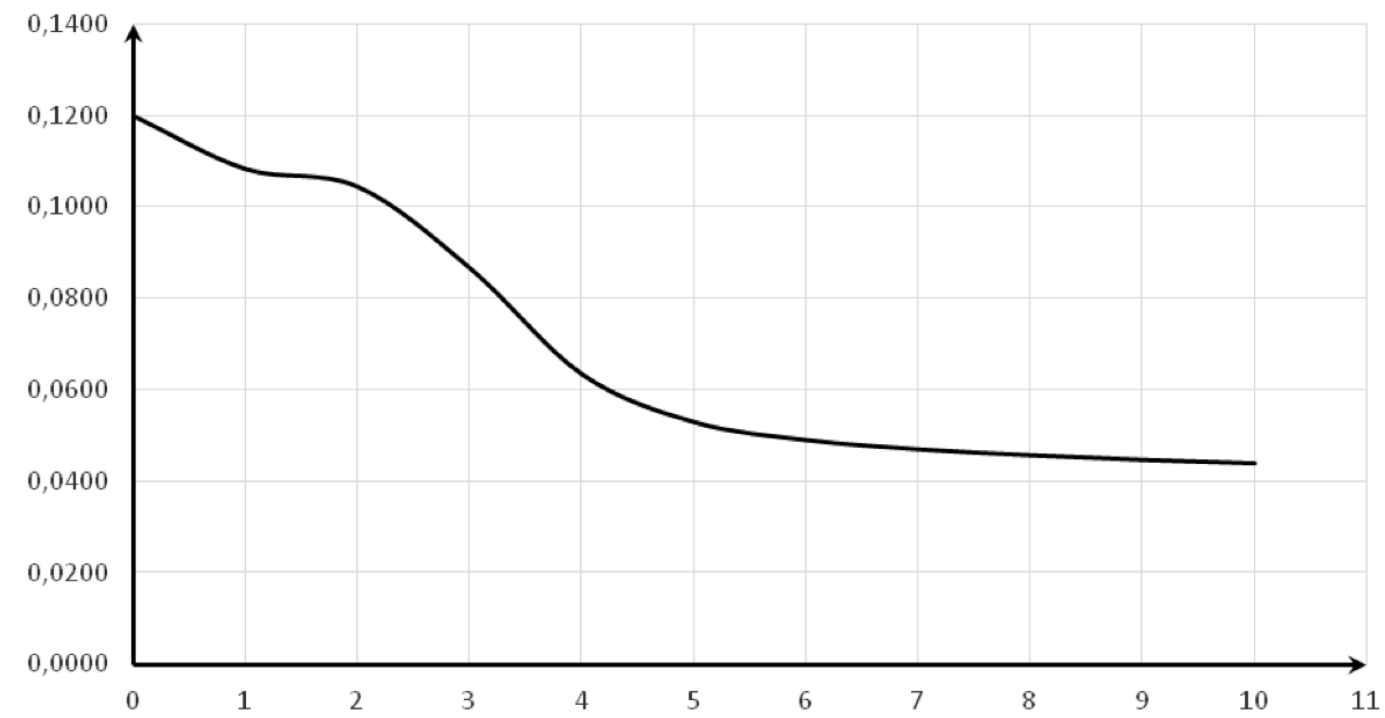

Figure 14: Dependence of WACC on L. 
One can see (Figures 3-14) that $W A C C(L)$ is monotonically diminishing function. In spite of the fact that the rise in the cost of debt financing was assumed, and fairly significant, WACC is not growing with leverage.

In dependence of WACC $(\mathrm{L})$ a cupped zone (in the mathematical sense, $W A C C_{L^{2}}<0$ ) appears only, which more or less corresponds to the leverage level, at which the increase in the cost of debt capital begins (in our case, $L=2$ ).

Note that distortion of the WACC $(\mathrm{L})$ dependence is mostly determined by the function $k_{d}(L)$ (linear or quadratic) and by the factors at $(L-2)$ or $(L-2)^{2}$. Linear dependence of $k_{d}(L)$ distorts the WACC $(\mathrm{L})$ dependence less than square one, as well as the smaller factor $(0,01)$.

The change of the company life time (from 3 to 5 years), has a smaller effect, although a bigger life times may lead to a more substantial changes in WACC $(L)$ dependence. The reduction of a difference $k_{0}-k_{d}$ between $k_{0}$ and $k_{d}$ leads to an increase of effect.

The main conclusion that can be drawn from the obtained results is the following: the optimal capital structure in well known "tradeoff" theory is missing, contrary to hopes and expectations of its creators and supporters.

The question immediately appears: why this turned out to be possible, and how this can be? How can the weighted average cost of capital

1. $n=3 ; t=20 \% ; L=0,1,2, \ldots 10$

$k_{0}=24 \% ; \bar{k}_{d}=\left\{\begin{array}{c}0,07 ; \text { at } L \leq 2 \\ 0,07+0,01(L-2)^{2} ; \text { at } L>2\end{array}\right\}$
$W A C C=w_{e} k_{e}+w_{d} k_{d}(1-T)$,

not grow, if both $k_{d}$ and $k_{e}$ are growing ( $k_{e}$ is growing with leverage in accordance to (3) and $k_{d}$ is growing in accordance to our assumption)?

The answer will be received in the next paragraph, where we are investigating the dependence of equity cost $k_{e}$ on leverage $\mathrm{L}$ with the same assumptions about the risky of debt capital and growth as a consequence of its cost with the leverage.

\section{THE CAUSES OF ABSENCE OF THE OPTIMUM CAPITAL STRUCTURE IN TRADEOFF THEORY}

So, we will investigate the dependence of the equity cost $k_{e}$ on leverage $\mathrm{L}$ at the same assumptions about the risky of the debt and growth of its cost with leverage.

In the Modigliani-Miller theory equity cost $k_{e}$ always grows with leverage, as well as in Brusov - Filatova Orekhova theory. In the latter one, however, an abnormal effect, discovered by us, exists (Brusov et al. 2013): decreasing of equity cost $k_{e}$ with leverage L. This effect, which is absent in perpetuity ModiglianiMiller limit, takes place under account of finite lifetime of the company at tax on profits rate, which exceeds some value $T^{*}$. At some ratios between debt cost and equity capital cost the discovered effect takes place at tax on profits rate, existing in western countries and Russia. But this effect has been obtained under condition of a constant debt cost $k_{d}$. Let us see, how the growth of debt cost $k_{d}$ with leverage affects the equity cost $k_{e}$ dependence on leverage. We will consider the same cases as above for the calculations of dependences WACC $(\mathrm{L})$.

Table 13:

\begin{tabular}{|c|c||c|c|c|c|c|c|c|c|c|c|c|}
\hline $\mathbf{n}$ & $\mathbf{L}$ & $\mathbf{0}$ & $\mathbf{1}$ & $\mathbf{2}$ & $\mathbf{3}$ & $\mathbf{4}$ & $\mathbf{5}$ & $\mathbf{6}$ & $\mathbf{7}$ & $\mathbf{8}$ & $\mathbf{9}$ & $\mathbf{1 0}$ \\
\hline \hline $\mathbf{3}$ & $\mathbf{k d}$ & 0,07 & 0,07 & 0,07 & 0,08 & 0,11 & 0,16 & 0,23 & 0,32 & 0,43 & 0,56 & 0,71 \\
\hline $\mathbf{k 0}$ & $\mathbf{A}$ & 1,9813 & 2,0184 & 2,0311 & 2,0445 & 2,0703 & 2,1075 & 2,1520 & 2,1988 & 2,2438 & 2,2842 & 2,3186 \\
\hline $\mathbf{0 , 2 4}$ & $\mathbf{K e}$ & 0,2401 & 0,3997 & 0,5594 & 0,6861 & 0,7036 & 0,5581 & 0,2011 & $-0,4081$ & $-1,3075$ & $-2,5356$ & $-4,1333$ \\
\hline
\end{tabular}




\section{$\mathrm{Ke}(\mathrm{L})$}

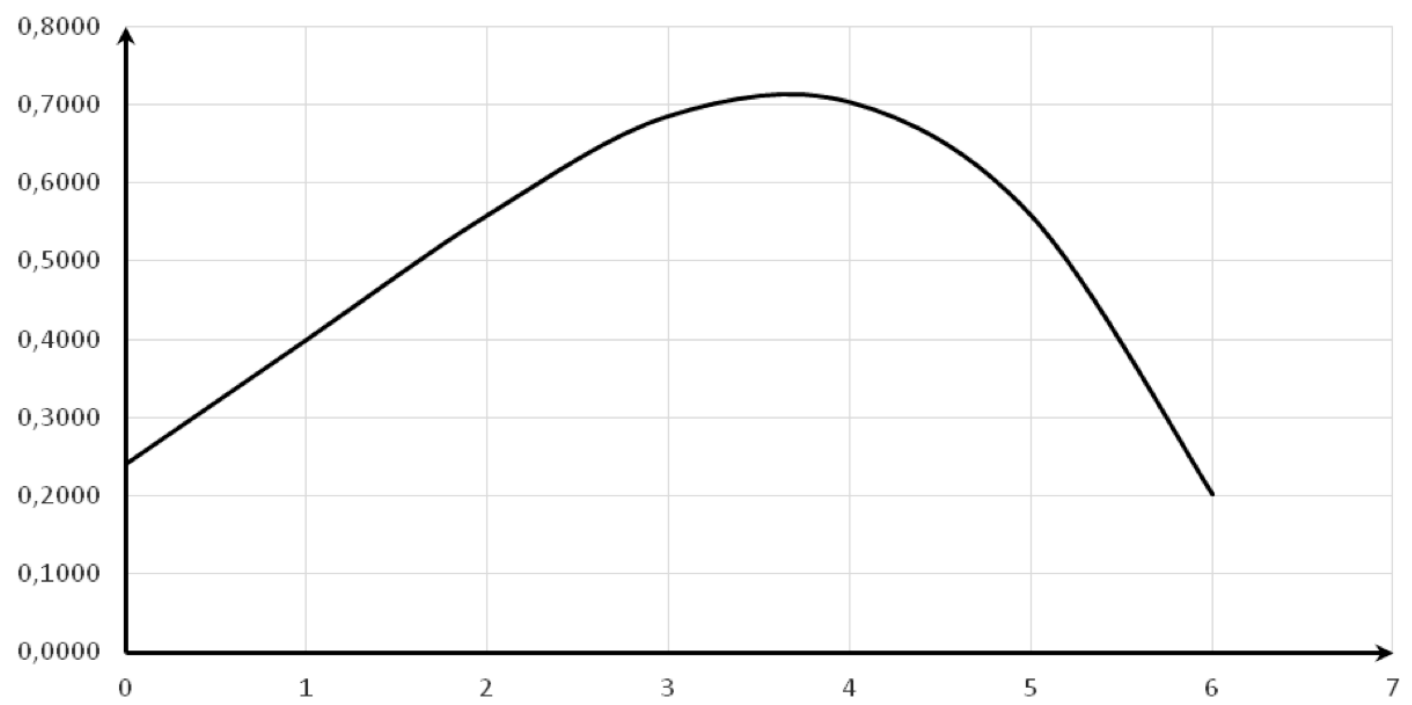

Figure 15: Dependence of $k_{e}$ on L.

2. $n=5 ; t=20 \% ; L=0,1,2, \ldots 10$

$k_{0}=24 \% ; \bar{k}_{d}=\left\{\begin{array}{c}0,07 ; a t L \leq 2 \\ 0,07+0,01(L-2)^{2} ; \text { at } L>2\end{array}\right\}$

Table 14:

\begin{tabular}{|c|c||c|c|c|c|c|c|c|c|c|c|c|}
\hline $\mathbf{n}$ & $\mathbf{L}$ & $\mathbf{0}$ & $\mathbf{1}$ & $\mathbf{2}$ & $\mathbf{3}$ & $\mathbf{4}$ & $\mathbf{5}$ & $\mathbf{6}$ & $\mathbf{7}$ & $\mathbf{8}$ & $\mathbf{9}$ & $\mathbf{1 0}$ \\
\hline \hline $\mathbf{5}$ & $\mathbf{k d}$ & 0,07 & 0,07 & 0,07 & 0,08 & 0,11 & 0,16 & 0,23 & 0,32 & 0,43 & 0,56 & 0,71 \\
\hline $\mathbf{k 0}$ & $\mathbf{A}$ & 2,7454 & 2,8265 & 2,8546 & 2,8835 & 2,9364 & 3,0080 & 3,0866 & 3,1605 & 3,2225 & 3,2703 & 3,3052 \\
\hline $\mathbf{0 , 2 4}$ & $\mathbf{K e}$ & 0,2400 & 0,3962 & 0,5524 & 0,6750 & 0,6897 & 0,5438 & 0,1966 & $-0,3892$ & $-1,2501$ & $-2,4267$ & $-3,9640$ \\
\hline
\end{tabular}

$\operatorname{Ke}(L)$

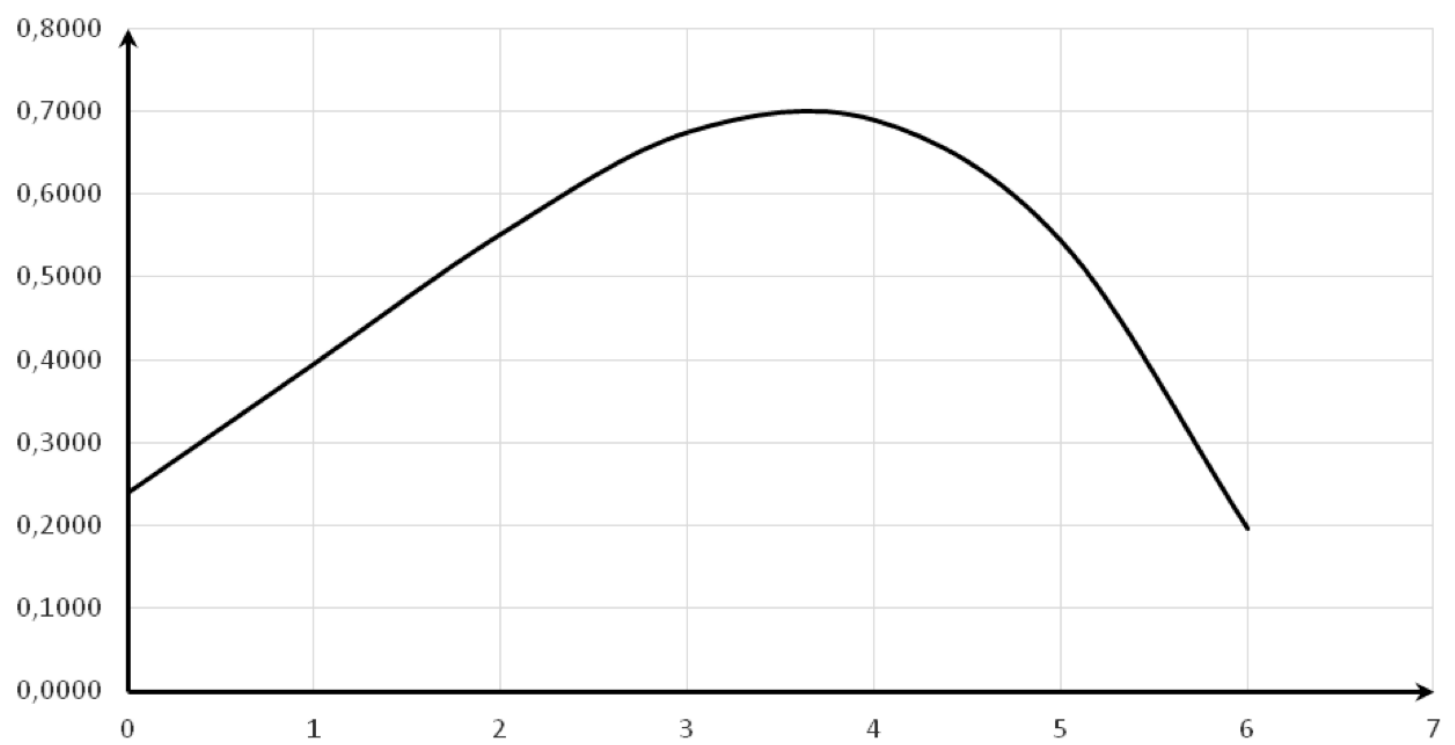

Figure 16: Dependence of $k_{e}$ on L. 
3. $n=3 ; t=20 \% ; L=0,1,2, \ldots 10$

$k_{0}=24 \% ; \bar{k}_{d}=\left\{\begin{array}{c}0,07 ; \text { at } L \leq 2 \\ 0,07+0,1(L-2)^{2} ; \text { at } L>2\end{array}\right\}$

Table 15:

\begin{tabular}{|c|c||c|c|c|c|c|c|c|c|c|c|c|}
\hline $\mathbf{n}$ & $\mathbf{L}$ & $\mathbf{0}$ & $\mathbf{1}$ & $\mathbf{2}$ & $\mathbf{3}$ & $\mathbf{4}$ & $\mathbf{5}$ & $\mathbf{6}$ & $\mathbf{7}$ & $\mathbf{8}$ & $\mathbf{9}$ & $\mathbf{1 0}$ \\
\hline \hline $\mathbf{3}$ & $\mathbf{k d}$ & 0,07 & 0,07 & 0,07 & 0,17 & 0,47 & 0,97 & 1,67 & 2,57 & 3,67 & 4,97 & 6,47 \\
\hline $\mathbf{k 0}$ & $\mathbf{A}$ & 1,9813 & 2,0184 & 2,0311 & 2,0996 & 2,2253 & 2,3170 & 2,3655 & 2,3904 & 2,4046 & 2,4137 & 2,4203 \\
\hline $\mathbf{0 , 2 4}$ & $\mathbf{K e}$ & 0,2401 & 0,3997 & 0,5594 & 0,4003 & $-0,6760$ & $-3,0339$ & $-7,1136$ & $-13,4098$ & $-22,4140$ & $-34,6126$ & $-50,4888$ \\
\hline
\end{tabular}

\section{$\operatorname{Ke}(L)$}

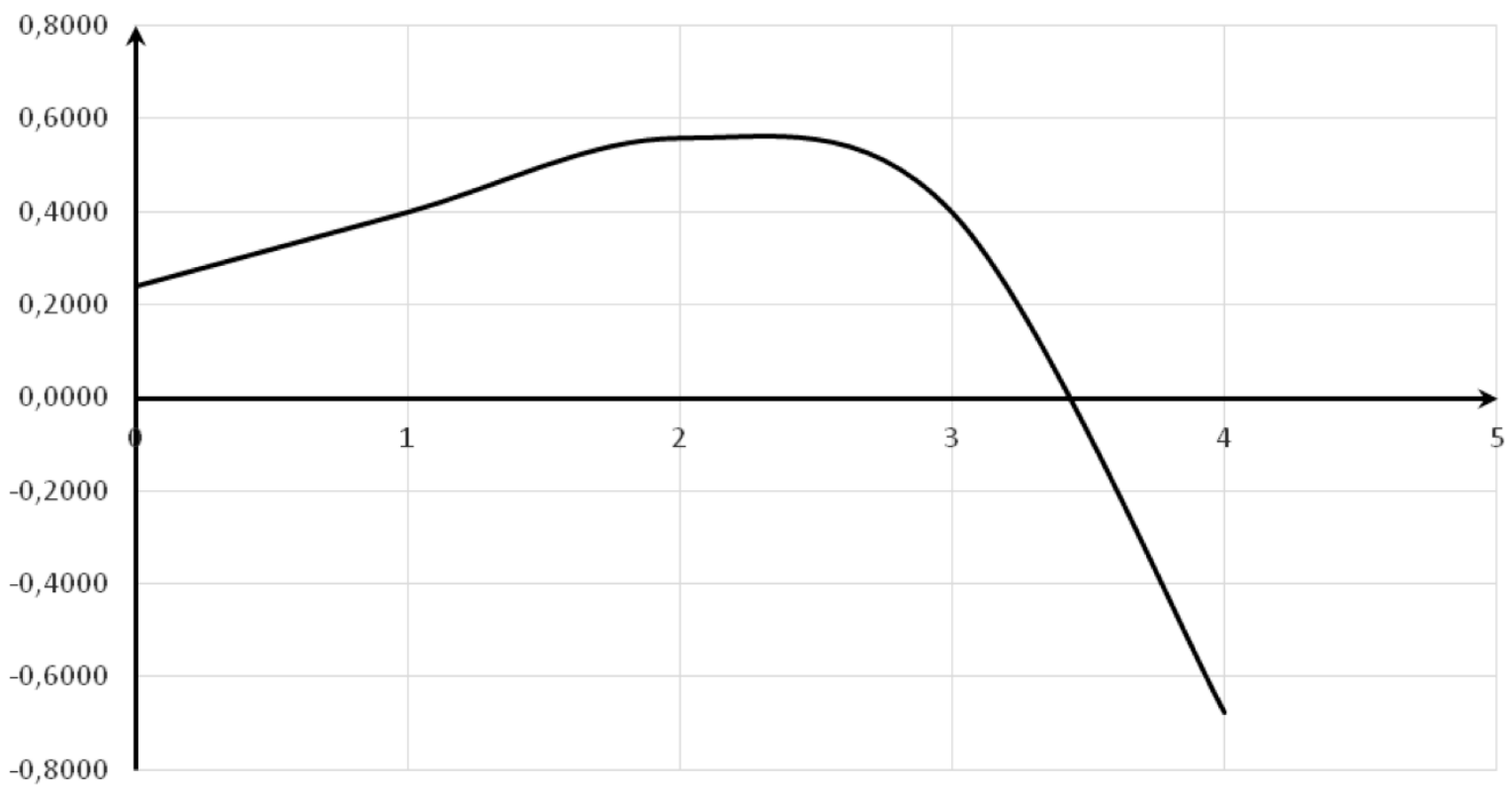

Figure 17: Dependence of $k_{e}$ on L.

4. $n=5 ; t=20 \% ; L=0,1,2, \ldots 10$

$k_{0}=24 \% ; \bar{k}_{d}=\left\{\begin{array}{c}0,07 ; \text { at } L \leq 2 \\ 0,07+0,1(L-2)^{2} ; \text { at } L>2\end{array}\right\}$

Table 16:

\begin{tabular}{|c|c||c|c|c|c|c|c|c|c|c|c|c|}
\hline $\mathbf{n}$ & $\mathbf{L}$ & $\mathbf{0}$ & $\mathbf{1}$ & $\mathbf{2}$ & $\mathbf{3}$ & $\mathbf{4}$ & $\mathbf{5}$ & $\mathbf{6}$ & $\mathbf{7}$ & $\mathbf{8}$ & $\mathbf{9}$ & $\mathbf{1 0}$ \\
\hline \hline $\mathbf{5}$ & $\mathbf{k d}$ & 0,07 & 0,07 & 0,07 & 0,17 & 0,47 & 0,97 & 1,67 & 2,57 & 3,67 & 4,97 & 6,47 \\
\hline $\mathbf{k 0}$ & $\mathbf{A}$ & 2,7454 & 2,8265 & 2,8546 & 2,9893 & 3,1801 & 3,2724 & 3,3084 & 3,3265 & 3,3387 & 3,3479 & 3,3554 \\
\hline $\mathbf{0 , 2 4}$ & $\mathbf{K e}$ & 0,2400 & 0,3962 & 0,5524 & 0,3926 & $-0,6408$ & $-2,9184$ & $-6,9268$ & $-13,1658$ & $-22,1224$ & $-34,2784$ & $-50,1142$ \\
\hline
\end{tabular}




\section{$\operatorname{Ke}(L)$}

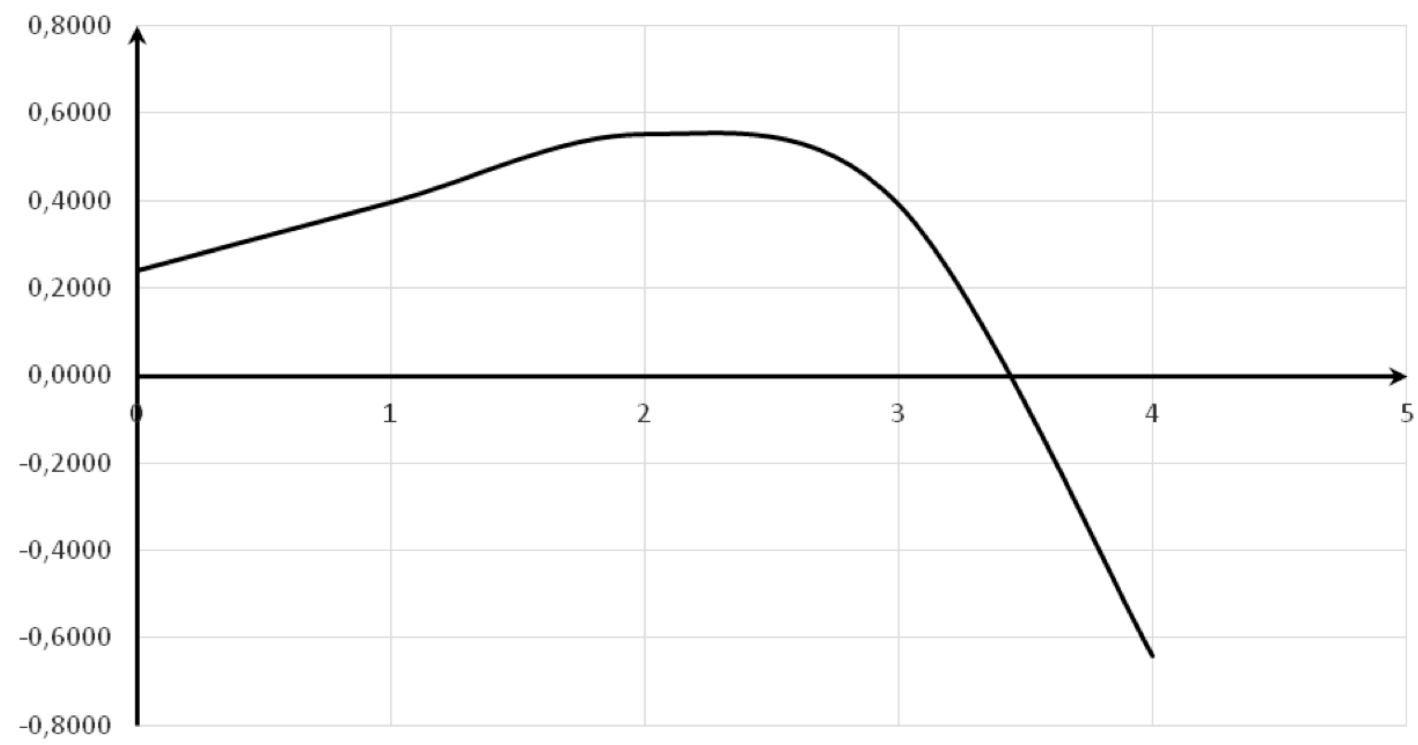

Figure 18: Dependence of $k_{e}$ on L.

5. $n=3 ; t=20 \% ; L=0,1,2, \ldots 10$

$k_{0}=24 \% ; \bar{k}_{d}=\left\{\begin{array}{c}0,07 ; \text { at } L \leq 2 \\ 0,07+0,01(L-2) ; \text { at } L>2\end{array}\right\}$

Table 17:

\begin{tabular}{|c|c|c|c|c|c|c|c|c|c|c|c|c|}
\hline $\mathbf{n}$ & $\mathbf{L}$ & $\mathbf{0}$ & $\mathbf{1}$ & $\mathbf{2}$ & $\mathbf{3}$ & $\mathbf{4}$ & $\mathbf{5}$ & $\mathbf{6}$ & $\mathbf{7}$ & $\mathbf{8}$ & $\mathbf{9}$ & $\mathbf{1 0}$ \\
\hline \hline $\mathbf{3}$ & $\mathbf{k d}$ & 0,07 & 0,07 & 0,07 & 0,08 & 0,09 & 0,1 & 0,11 & 0,12 & 0,13 & 0,14 & 0,15 \\
\hline $\mathbf{k 0}$ & $\mathbf{A}$ & 1,9813 & 2,0184 & 2,0311 & 2,0445 & 2,0563 & 2,0670 & 2,0770 & 2,0865 & 2,0957 & 2,1044 & 2,1129 \\
\hline $\mathbf{0 , 2 4}$ & $\mathbf{K e}$ & 0,2401 & 0,3997 & 0,5594 & 0,6861 & 0,7913 & 0,8730 & 0,9353 & 0,9767 & 0,9976 & 0,9982 & 0,9787 \\
\hline
\end{tabular}

\section{$\operatorname{Ke}(L)$}

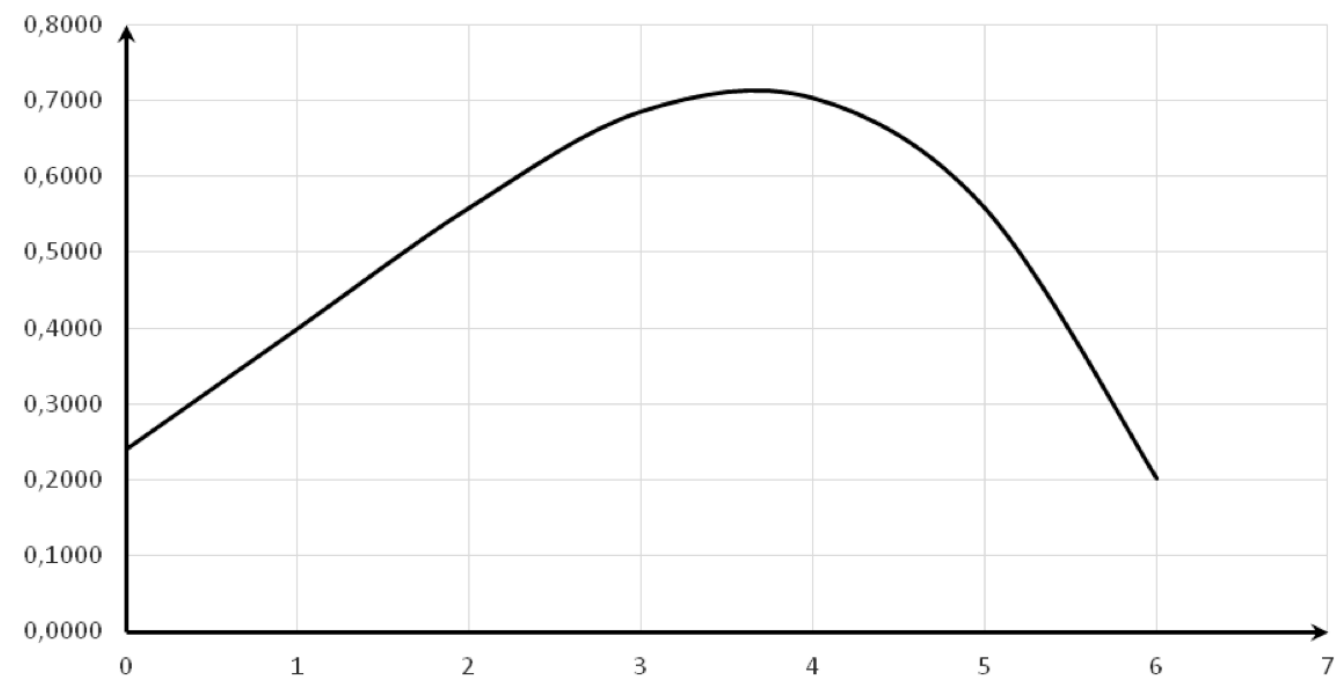

Figure 19: Dependence of $k_{e}$ on L. 
6. $n=5 ; t=20 \% ; L=0,1,2, \ldots 10$

$k_{0}=24 \% ; \bar{k}_{d}=\left\{\begin{array}{c}0,07 ; \text { at } L \leq 2 \\ 0,07+0,01(L-2) ; \text { at } L>2\end{array}\right\}$

Table 18:

\begin{tabular}{|c|c||c|c|c|c|c|c|c|c|c|c|c|}
\hline $\mathbf{n}$ & $\mathbf{L}$ & $\mathbf{0}$ & $\mathbf{1}$ & $\mathbf{2}$ & $\mathbf{3}$ & $\mathbf{4}$ & $\mathbf{5}$ & $\mathbf{6}$ & $\mathbf{7}$ & $\mathbf{8}$ & $\mathbf{9}$ & $\mathbf{1 0}$ \\
\hline \hline $\mathbf{5}$ & $\mathbf{k d}$ & 0,07 & 0,07 & 0,07 & 0,08 & 0,09 & 0,1 & 0,11 & 0,12 & 0,13 & 0,14 & 0,15 \\
\hline $\mathbf{k 0}$ & $\mathbf{A}$ & 2,7454 & 2,8265 & 2,8546 & 2,8835 & 2,9083 & 2,9305 & 2,9511 & 2,9702 & 2,9883 & 3,0054 & 3,0216 \\
\hline $\mathbf{0 , 2 4}$ & $\mathbf{K e}$ & 0,2400 & 0,3962 & 0,5524 & 0,6750 & 0,7759 & 0,8555 & 0,9143 & 0,9525 & 0,9706 & 0,9689 & 0,9477 \\
\hline
\end{tabular}

\section{$\operatorname{Ke}(L)$}

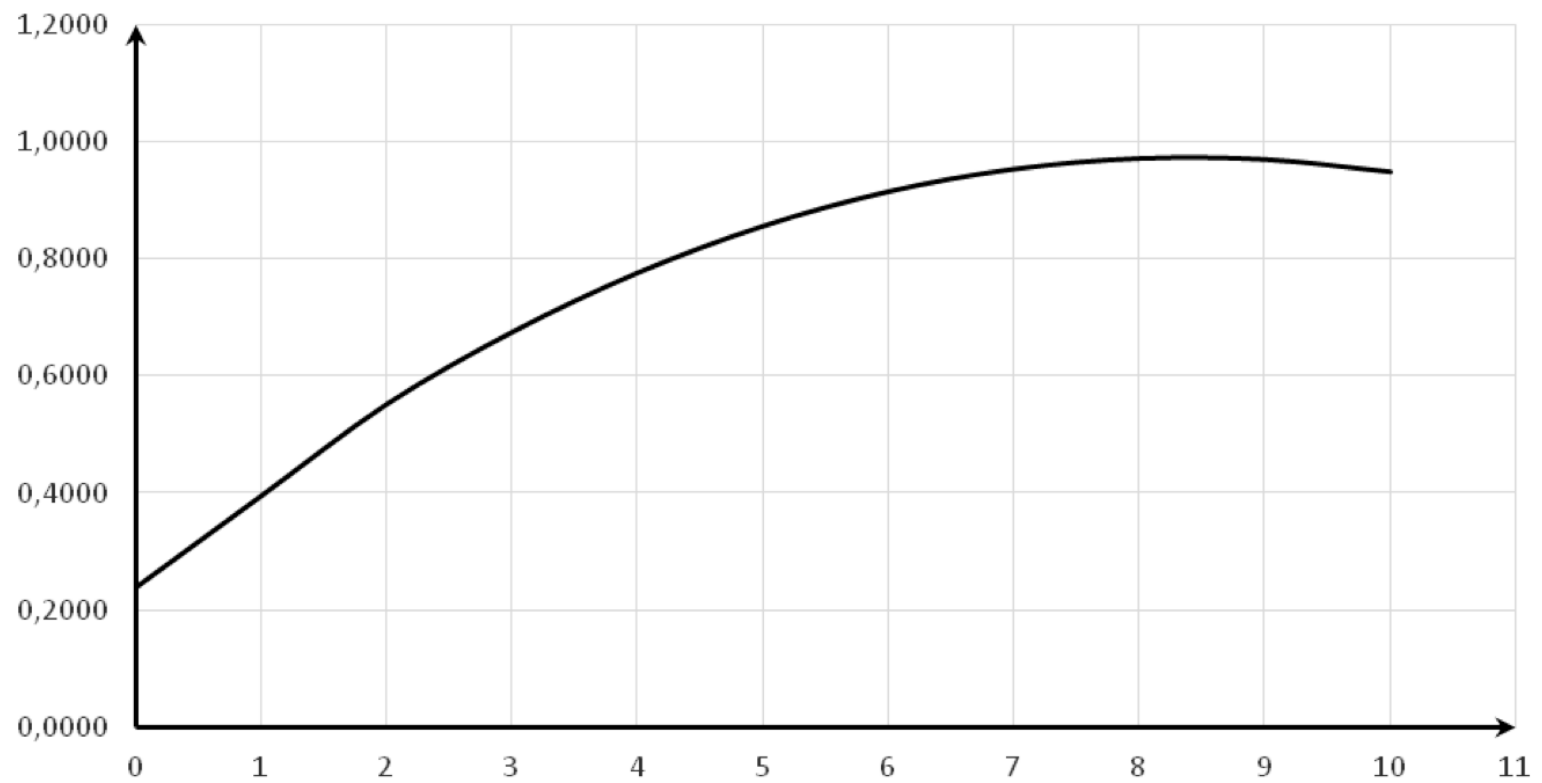

Figure 20: Dependence of $k_{e}$ on L.

7. $n=3 ; t=20 \% ; L=0,1,2, \ldots 10$

$k_{0}=24 \% ; \bar{k}_{d}=\left\{\begin{array}{c}0,07 ; \text { at } L \leq 2 \\ 0,07+0,1(L-2) ; \text { at } L>2\end{array}\right\}$

Table 19:

\begin{tabular}{|c|c||c|c|c|c|c|c|c|c|c|c|c|}
\hline $\mathbf{n}$ & $\mathbf{L}$ & $\mathbf{0}$ & $\mathbf{1}$ & $\mathbf{2}$ & $\mathbf{3}$ & $\mathbf{4}$ & $\mathbf{5}$ & $\mathbf{6}$ & $\mathbf{7}$ & $\mathbf{8}$ & $\mathbf{9}$ & $\mathbf{1 0}$ \\
\hline \hline $\mathbf{3}$ & $\mathbf{k d}$ & 0,07 & 0,07 & 0,07 & 0,17 & 0,27 & 0,37 & 0,47 & 0,57 & 0,67 & 0,77 & 0,87 \\
\hline $\mathbf{k 0}$ & $\mathbf{A}$ & 1,9813 & 2,0184 & 2,0311 & 2,0996 & 2,1580 & 2,2060 & 2,2450 & 2,2768 & 2,3028 & 2,3242 & 2,3420 \\
\hline $\mathbf{0 , 2 4}$ & $\mathbf{K e}$ & 0,2401 & 0,3997 & 0,5594 & 0,4003 & 0,0594 & $-0,4542$ & $-1,1348$ & $-1,9792$ & $-2,9855$ & $-4,1526$ & $-5,4797$ \\
\hline
\end{tabular}




\section{$\operatorname{Ke}(L)$}

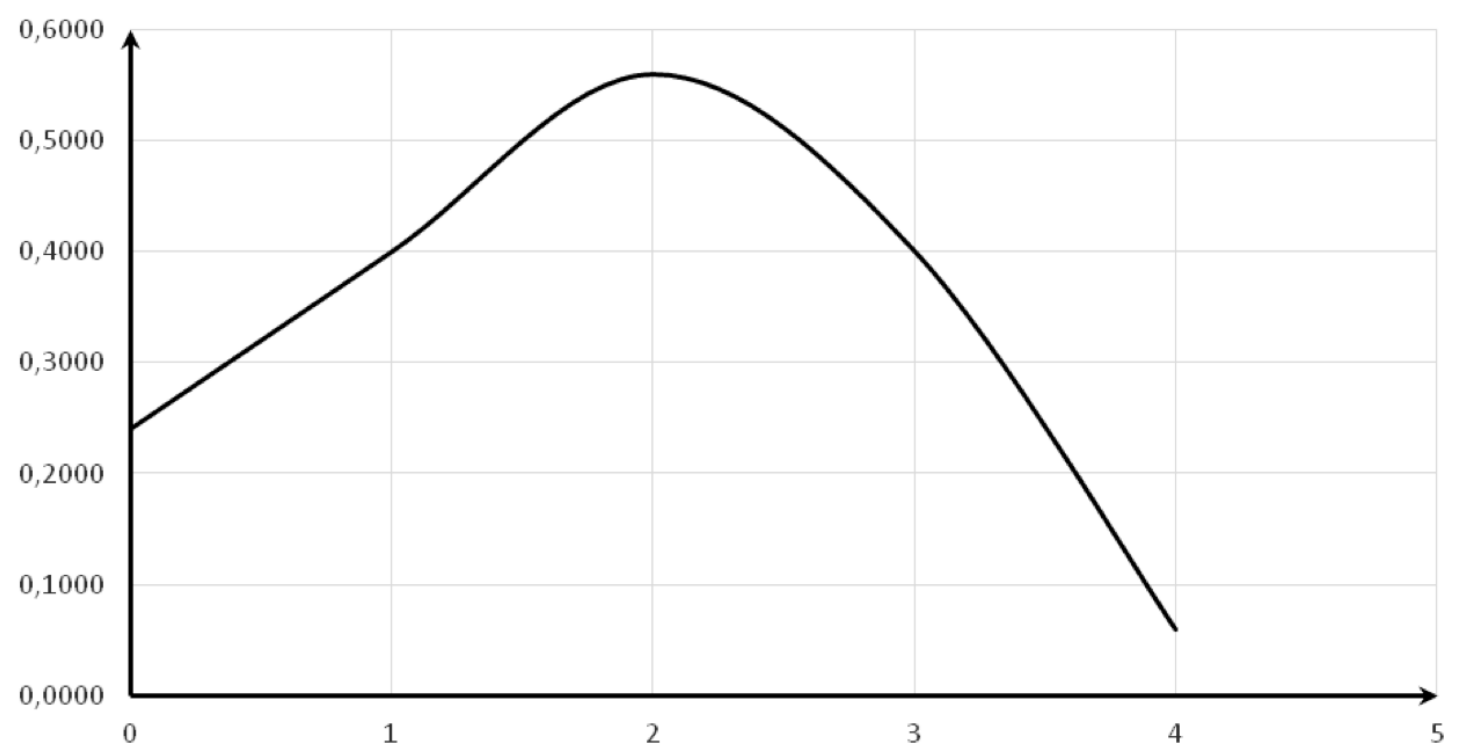

Figure 21: Dependence of $k_{e}$ on L.

8. $n=5 ; t=20 \% ; L=0,1,2, \ldots 10$

$k_{0}=24 \% ; \bar{k}_{d}=\left\{\begin{array}{c}0,07 ; \text { at } L \leq 2 \\ 0,07+0,1(L-2) ; \text { at } L>2\end{array}\right\}$

\section{Table 20:}

\begin{tabular}{|c|c||c|c|c|c|c|c|c|c|c|c|c|}
\hline $\mathbf{n}$ & $\mathbf{L}$ & $\mathbf{0}$ & $\mathbf{1}$ & $\mathbf{2}$ & $\mathbf{3}$ & $\mathbf{4}$ & $\mathbf{5}$ & $\mathbf{6}$ & $\mathbf{7}$ & $\mathbf{8}$ & $\mathbf{9}$ & $\mathbf{1 0}$ \\
\hline \hline $\mathbf{5}$ & $\mathbf{k d}$ & 0,07 & 0,07 & 0,07 & 0,17 & 0,27 & 0,37 & 0,47 & 0,57 & 0,67 & 0,77 & 0,87 \\
\hline $\mathbf{k 0}$ & $\mathbf{A}$ & 2,7454 & 2,8265 & 2,8546 & 2,9893 & 3,0902 & 3,1634 & 3,2164 & 3,2553 & 3,2843 & 3,3063 & 3,3232 \\
\hline $\mathbf{0 , 2 4}$ & $\mathbf{K e}$ & 0,2400 & 0,3962 & 0,5524 & 0,3926 & 0,0624 & $-0,4304$ & $-1,0822$ & $-1,8920$ & $-2,8596$ & $-3,9853$ & $-5,2693$ \\
\hline
\end{tabular}

\section{$\operatorname{Ke}(\mathrm{L})$}

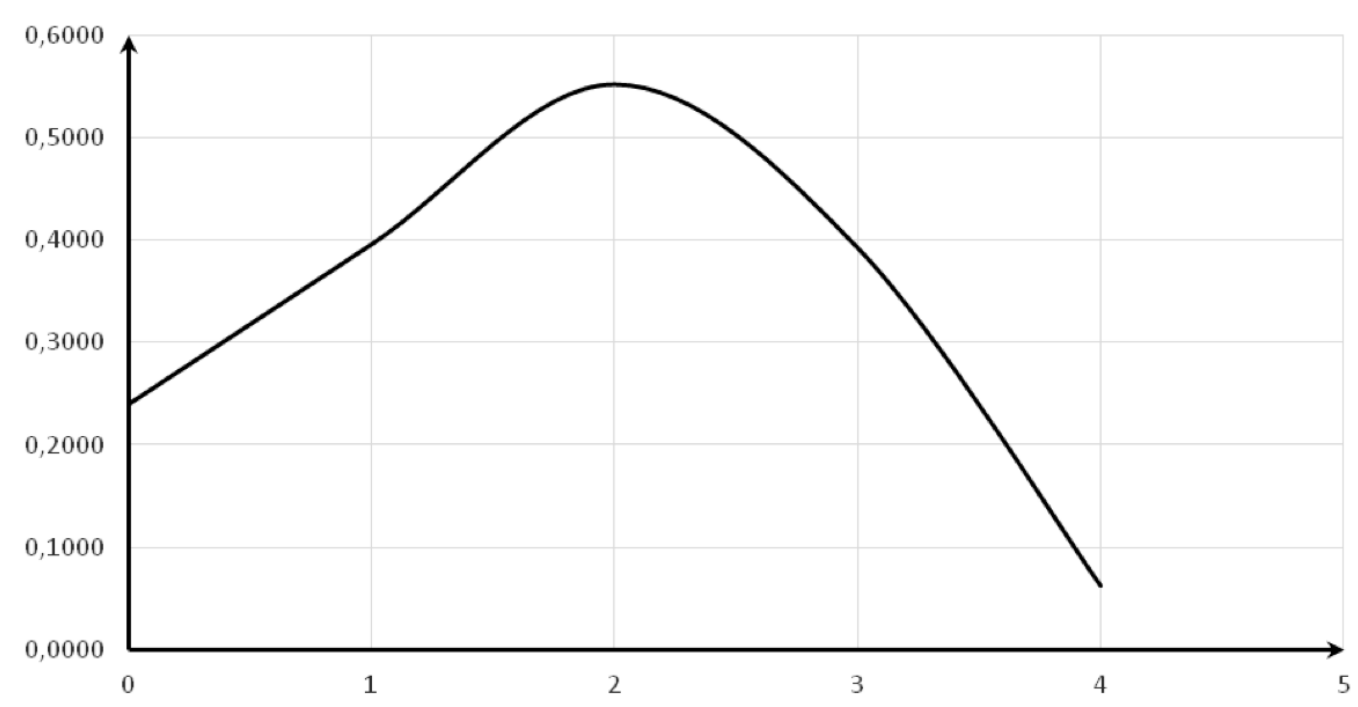

Figure 22: Dependence of $k_{e}$ on L. 
9. $n=3 ; t=20 \% ; L=0,1,2, \ldots 10$

$k_{0}=12 \% ; \bar{k}_{d}=\left\{\begin{array}{c}0,07 ; \text { at } L \leq 2 \\ 0,07+0,01(L-2)^{2} ; \text { at } L>2\end{array}\right\}$

Table 21:

\begin{tabular}{|c|c||c|c|c|c|c|c|c|c|c|c|c|}
\hline $\mathbf{n}$ & $\mathbf{L}$ & $\mathbf{0}$ & $\mathbf{1}$ & $\mathbf{2}$ & $\mathbf{3}$ & $\mathbf{4}$ & $\mathbf{5}$ & $\mathbf{6}$ & $\mathbf{7}$ & $\mathbf{8}$ & $\mathbf{9}$ & $\mathbf{1 0}$ \\
\hline \hline $\mathbf{3}$ & $\mathbf{k d}$ & 0,07 & 0,07 & 0,07 & 0,08 & 0,11 & 0,16 & 0,23 & 0,32 & 0,43 & 0,56 & 0,71 \\
\hline $\mathbf{k 0}$ & $\mathbf{A}$ & 2,4018 & 2,4468 & 2,4621 & 2,4785 & 2,5098 & 2,5548 & 2,6087 & 2,6655 & 2,7200 & 2,7690 & 2,8107 \\
\hline $\mathbf{0 , 1 2}$ & $\mathbf{K e}$ & 0,1200 & 0,1626 & 0,2051 & 0,2157 & 0,1222 & $-0,1307$ & $-0,5904$ & $-1,2998$ & $-2,2963$ & $-3,6202$ & $-5,3133$ \\
\hline
\end{tabular}

$\operatorname{Ke}(L)$

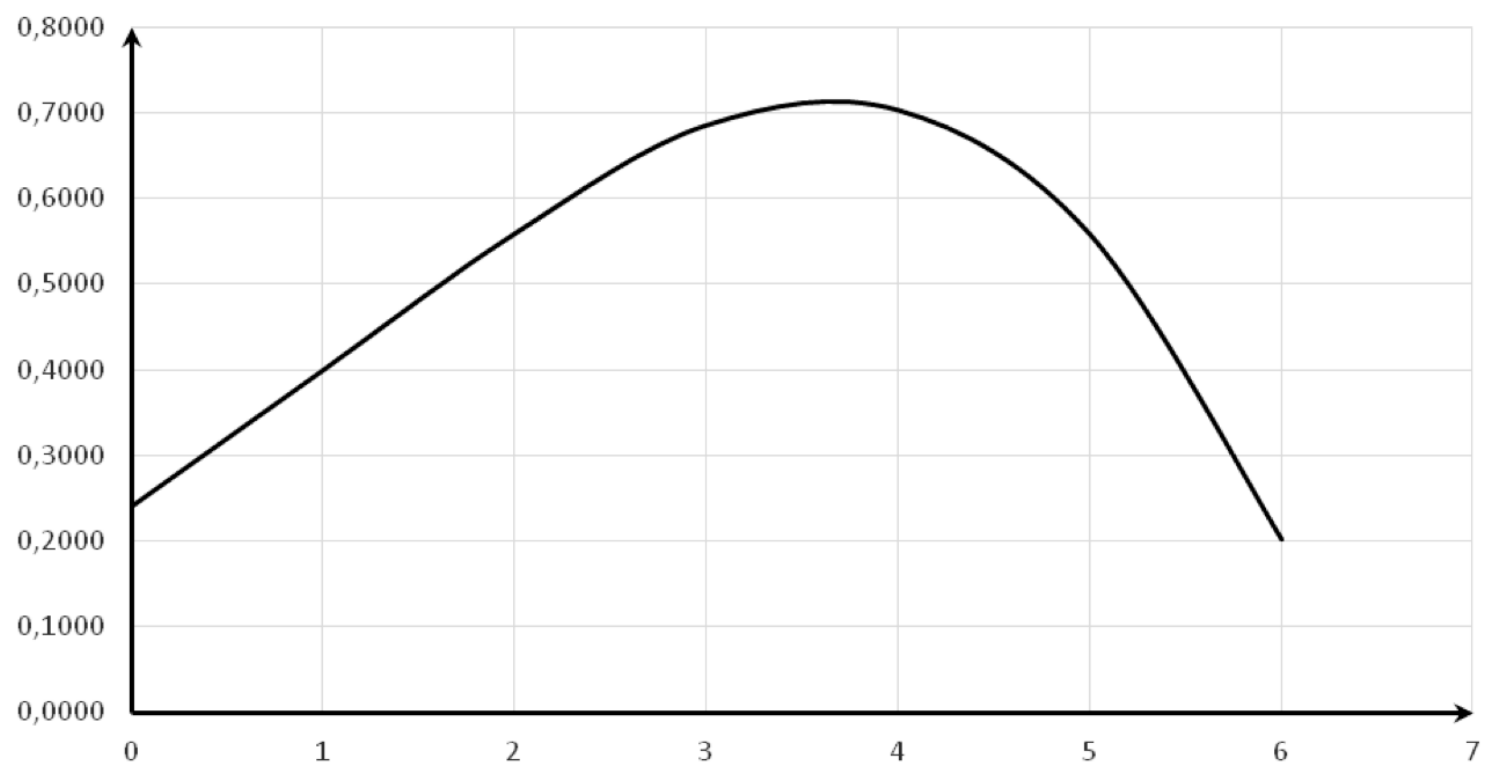

Figure 23: Dependence of $k_{e}$ on L.

10. $n=5 ; t=20 \% ; L=0,1,2, \ldots 10$

$k_{0}=12 \% ; \bar{k}_{d}=\left\{\begin{array}{c}0,07 ; \text { at } L \leq 2 \\ 0,07+0,01(L-2)^{2} ; \text { at } L>2\end{array}\right\}$

Table 22:

\begin{tabular}{|c|c||c|c|c|c|c|c|c|c|c|c|c|}
\hline $\mathbf{n}$ & $\mathbf{L}$ & $\mathbf{0}$ & $\mathbf{1}$ & $\mathbf{2}$ & $\mathbf{3}$ & $\mathbf{4}$ & $\mathbf{5}$ & $\mathbf{6}$ & $\mathbf{7}$ & $\mathbf{8}$ & $\mathbf{9}$ & $\mathbf{1 0}$ \\
\hline \hline $\mathbf{5}$ & $\mathbf{k d}$ & 0,07 & 0,07 & 0,07 & 0,08 & 0,11 & 0,16 & 0,23 & 0,32 & 0,43 & 0,56 & 0,71 \\
\hline $\mathbf{k 0}$ & $\mathbf{A}$ & 3,6048 & 3,7113 & 3,7482 & 3,7862 & 3,8556 & 3,9496 & 4,0528 & 4,1498 & 4,2312 & 4,2940 & 4,3399 \\
\hline $\mathbf{0 , 1 2}$ & $\mathbf{K e}$ & 0,1200 & 0,1607 & 0,2014 & 0,2100 & 0,1152 & $-0,1352$ & $-0,5829$ & $-1,2677$ & $-2,2267$ & $-3,5020$ & $-5,1389$ \\
\hline
\end{tabular}


$\operatorname{Ke}(L)$

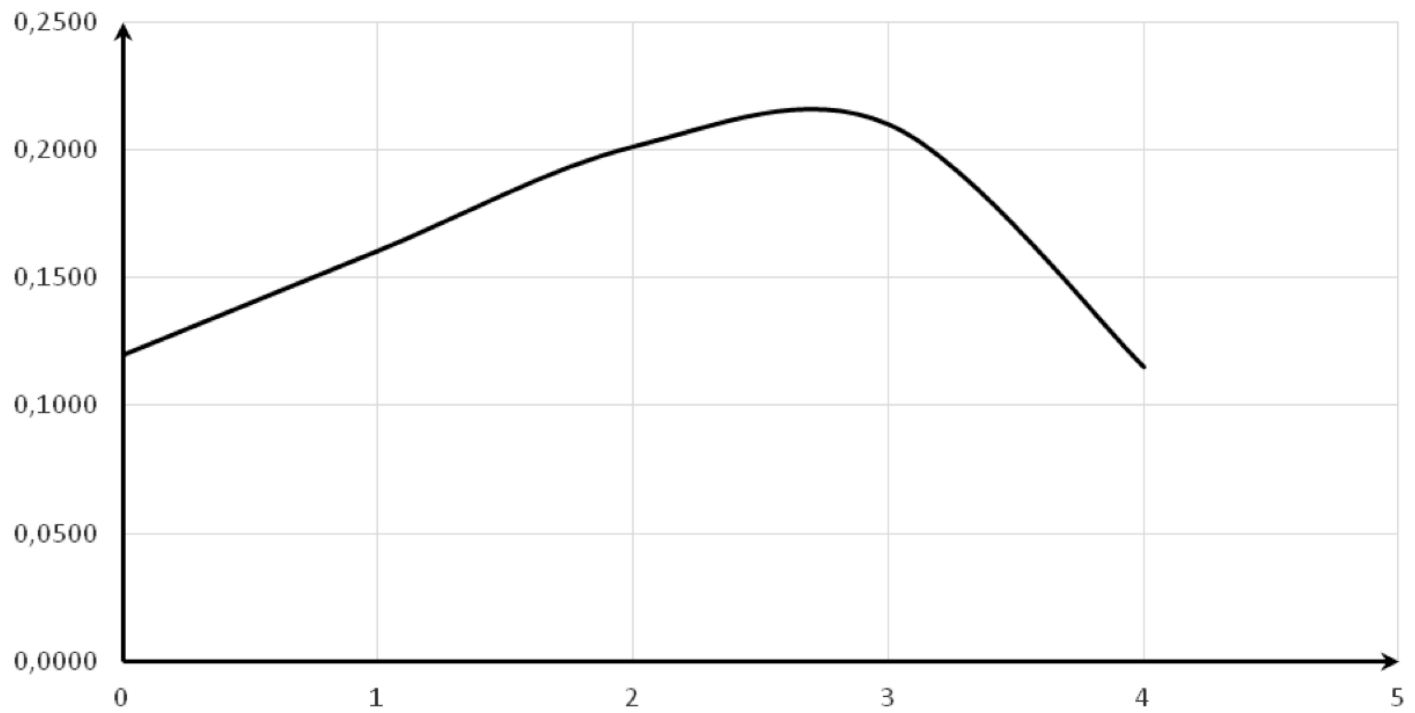

Figure 24: Dependence of $k_{e}$ on L.

11. $n=3 ; t=20 \% ; L=0,1,2, \ldots 10$

$k_{0}=12 \% ; \bar{k}_{d}=\left\{\begin{array}{c}0,07 ; a t L \leq 2 \\ 0,07+0,1(L-2)^{2} ; \text { at } L>2\end{array}\right\}$

Table 23:

\begin{tabular}{|c|c||c|c|c|c|c|c|c|c|c|c|c|}
\hline $\mathbf{n}$ & $\mathbf{L}$ & $\mathbf{0}$ & $\mathbf{1}$ & $\mathbf{2}$ & $\mathbf{3}$ & $\mathbf{4}$ & $\mathbf{5}$ & $\mathbf{6}$ & $\mathbf{7}$ & $\mathbf{8}$ & $\mathbf{9}$ & $\mathbf{1 0}$ \\
\hline \hline $\mathbf{3}$ & $\mathbf{k d}$ & 0,07 & 0,07 & 0,07 & 0,17 & 0,47 & 0,97 & 1,67 & 2,57 & 3,67 & 4,97 & 6,47 \\
\hline $\mathbf{k 0}$ & $\mathbf{A}$ & 2,4018 & 2,4468 & 2,4621 & 2,5452 & 2,6976 & 2,8087 & 2,8676 & 2,8978 & 2,9150 & 2,9260 & 2,9340 \\
\hline $\mathbf{0 , 1 2}$ & $\mathbf{K e}$ & 0,1200 & 0,1626 & 0,2051 & $-0,0601$ & $-1,2286$ & $-3,6778$ & $-7,8553$ & $-14,2512$ & $-23,3566$ & $-35,6572$ & $-51,6356$ \\
\hline
\end{tabular}

$\operatorname{Ke}(\mathrm{L})$

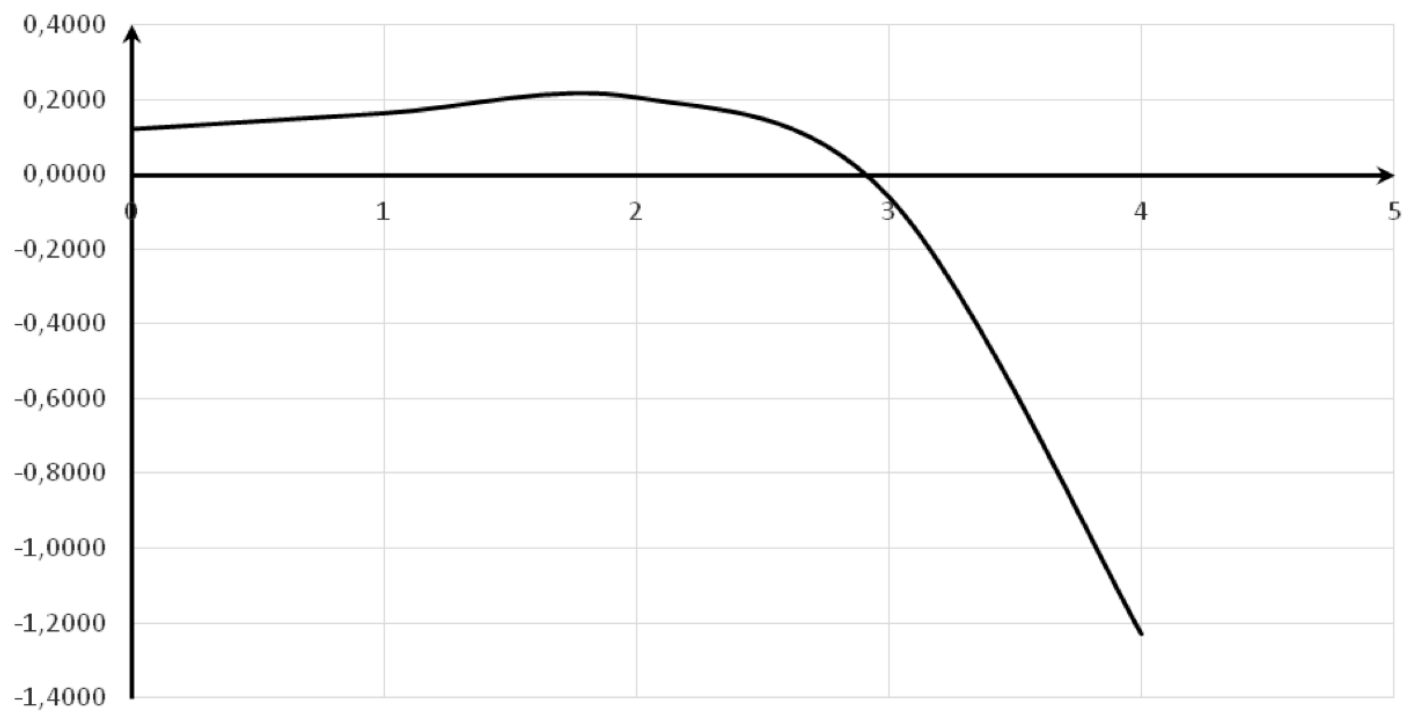

Figure 25: Dependence of $k_{e}$ on L. 
12. $n=5 ; t=20 \% ; L=0,1,2, \ldots 10$

$k_{0}=12 \% ; \bar{k}_{d}=\left\{\begin{array}{c}0,07 ; \text { at } L \leq 2 \\ 0,07+0,1(L-2)^{2} ; \text { at } L>2\end{array}\right\}$

Table 24:

\begin{tabular}{|c|c||c|c|c|c|c|c|c|c|c|c|c|}
\hline $\mathbf{n}$ & $\mathbf{L}$ & $\mathbf{0}$ & $\mathbf{1}$ & $\mathbf{2}$ & $\mathbf{3}$ & $\mathbf{4}$ & $\mathbf{5}$ & $\mathbf{6}$ & $\mathbf{7}$ & $\mathbf{8}$ & $\mathbf{9}$ & $\mathbf{1 0}$ \\
\hline \hline $\mathbf{5}$ & $\mathbf{k d}$ & 0,07 & 0,07 & 0,07 & 0,17 & 0,47 & 0,97 & 1,67 & 2,57 & 3,67 & 4,97 & 6,47 \\
\hline $\mathbf{k 0}$ & $\mathbf{A}$ & 3,6048 & 3,7113 & 3,7482 & 3,9250 & 4,1755 & 4,2968 & 4,3440 & 4,3678 & 4,3838 & 4,3959 & 4,4058 \\
\hline $\mathbf{0 , 1 2}$ & $\mathbf{K e}$ & 0,1200 & 0,1607 & 0,2014 & $-0,0615$ & $-1,1876$ & $-3,5634$ & $-7,6740$ & $-14,0175$ & $-23,0784$ & $-35,3389$ & $-51,2792$ \\
\hline
\end{tabular}

\section{$\operatorname{Ke}(\mathrm{L})$}

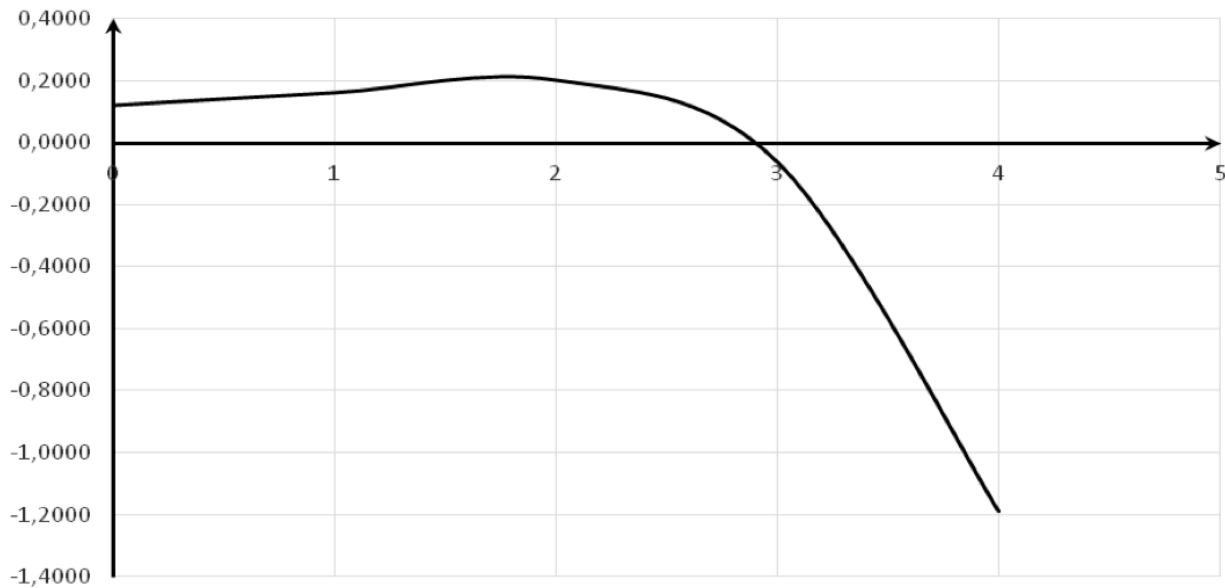

Figure 26: Dependence of $k_{e}$ on L.

An analysis of the obtained results (Figure 15-26) leads to the following conclusions.

Under the turning on the growth of debt cost $k_{d}$ with leverage, the dependence of equity cost $k_{e}$ on leverage is undergoing significant changes. The linear growth of equity cost $k_{e}$ at low leverage level is changed by its fall, starting from some value $L_{0}$. The $L_{0}$ value sometimes exactly correlates with the starting point of $k_{d}$ growth with leverage $(L=2)$, but sometimes takes values which are significantly higher (up to $L_{0}=8.5$ ).

The speed of decreasing of equity cost $k_{e}$ with leverage increases with increasing growth factor of debt cost $k_{d}$ as well as under the transition to quadratic growth. This is especially noticeable in the case 6 , where there is a $k_{e}$ growing, up to the leverage level $L_{0}=8.5$.

So, we come to the conclusion that the increase in the cost of debt capital $k_{d}$ with leverage leads to the decrease of equity cost $k_{e}$ with leverage, starting with some value $L_{0}$. This is the cause of the absence of weighted average capital cost growth with leverage at all its values.

Note, that the results remain qualitatively the same if we use any other dependences of $k_{d}$ on leverage. For example, for the case of exponential growing of $k_{d}$ with leverage

$$
\begin{aligned}
& n=5 ; t=20 \% ; L=0,1,2, \ldots 6, \\
& k_{0}=22 \% ; \bar{k}_{d}=\left\{\begin{array}{c}
0,12 ; \text { at } L \leq 1 \\
0,12+0,01 \cdot 3^{L-1} ; \text { at } L>1
\end{array}\right\}
\end{aligned}
$$

one gets the following dependence of $k_{e}, k_{d}$ and WACC on leverage (Figure 27).

So, the conclusions made are independent of rate of growing of $k_{d}$ with leverage.

\section{CONCLUSION}

The analysis of well-known tradeoff theory, conducting with the help of modern theory of capital 


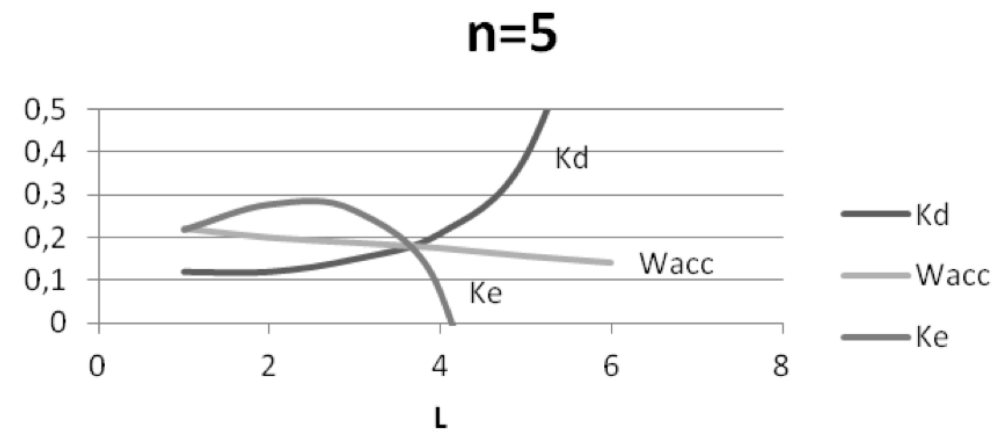

Figure 27: Dependence of $k_{d}, k_{d}$ and WACC on leverage.

structure and capital cost by Brusov-Filatova-Orekhova (BFO theory), has shown that that suggestion of risky debt financing (and growing credit rate near the bankruptcy) in opposite to waiting result does not lead to growing of WACC, which still decreases with leverage. This means the absence of minimum in the dependence of WACC on leverage as well as the absence of maximum in the dependence of capitalization $V$ on leverage. Thus, it seems that the optimal capital structure is absent in famous tradeoff theory. The explanation to this fact has been done within the same Brusov-Filatova-Orekhova theory by study the dependence of the equity cost $k_{e}$ on leverage. It turned out that the growth of debt cost $k_{d}$ with leverage lead to decrease of equity cost $k_{e}$ with leverage, starting from some leverage level, which is higher than starting point of debt cost growth. This paradox conclusion gives the explanation of the absence of the optimal capital structure in the famous tradeoff theory. This means, that competition of benefits from using of debt financing and growth of financial distress cost (or a bankruptcy cost) are NOT balanced and hopes, that tradeoff theory gives us the optimal capital structure, unfortunately, do not realized.

The absence of the optimal capital structure in the tradeoff theory questioned the existence of an optimal capital structure of the company (but as authors have shown, the optimal capital structure for the investment still exists (Brusov et al. 2011b, 2011c)). In the search for the "golden fleece" one needs to switch to study of other mechanisms for formation of the capital structure of the company, different from ones considering in tradeoff theory.

\section{APPENDIX. THE MODIGLIANI-MILLER THEORY ASSUMPTIONS}

The most important assumptions of the ModiglianiMiller theory are as following.

1. Investors are behaving rationally and instantaneously see profit opportunity, inadequate investment risk. Therefore, the possibility of a stable situation of the arbitration, i.e. obtain the risk- free profit on the difference in prices for the same asset can not be kept any length of time - a reasonable investors quickly take advantage of it for their own purposes and equalize conditions in the market. This means that in a developed financial market, capital the same risk should be rewarded by the same rate of return.

2. Investment and financial market opportunities should be equally accessible to all categories of investors - whether institutional or individual investors, large or small, rapidly growing or stable, experienced or relatively inexperienced.

3. Transaction costs associated with funding, are very small. In practice, the magnitude of transaction costs is inversely proportional to the amount of finance involved, so this assumption is the more consistent with reality than the large sums involved: i.e. in attracting small amounts the transaction costs can be high, while, as in attracting large loans, as well as during placement of shares at a significant amount the transaction costs can be ignored.

4. Investors get money and provide funds to borrowers at risk-free rate. In all probability, this assumption is due to the fact that the lender seeks to protect himself by using one or other guarantees, pledge of assets, the right to pay claims on third parties, the treaty provisions restricting the freedom of the borrower to act to the detriment of the creditor. Lender's risk is really small, but its position can be considered risk-free with respect to the position of the borrower and, accordingly, should be rewarded by a risk-free rate of return. 
5. Companies have only two types of assets: riskfree lending and risk their own.

6. There is no possibility of bankruptcy, i.e., irrespective of what level to bring its financial leverage of the company-borrowers, bankruptcy is threatening them. Thus bankruptcy costs are absent.

7. There are no corporate taxes and taxes on personal income of investors. If the personal income tax can indeed be neglected, because the assets of the company separated from the assets of shareholders, the corporate income taxes should be considered in the development of more realistic theories (which was done by Modigliani and Miller in the second paper devoted to the capital structure.

8. Companies are in the same class of risky companies.

9. All financial flows are perpetuity.

10. Companies have the same information.

11. Management of the company maximizes the equity of the company.

\section{REFERENCES}

Peter Brusov, Tatiana Filatova, Natali Orehova, 2013. Modern corporative finance and investment, KNORUS, p. 540.

Modigliani F., Miller M. 1958. The Cost of Capital, Corporate Finance and the Theory of Investment // American Economic Review. Vol. 48. № 4. P. 261-297.

Modigliani F., Miller M. 1963. Corporate Income Taxes and the Cost of Capital: A Correction // American Economic Review. Vol. 53. № 3. P. 147-175

Modigliani F., Miller M. 1966. Some estimates of the Cost of Capital to the Electric Utility Industry 1954-1957 // American Economic Review. P. 261-297.

Brusov P., Filatova T., Orehova N., Brusova N. 2011a. Weighted average cost of capital in the theory of Modigliani-Miller, modified for a finite life-time company. Applied Financial Economics. V. 21(11). P. 815-824. http://dx.doi.org/10.1080/09603107.2010.537635

Brusov P., Filatova T., Orehova N., Brusov P.P., Brusova N. 2011b. From Modigliani-Miller to general theory of capital cost and capital structure of the company. Research Journal of Economics, Business and ICT. V. 2. P. 16-21.
Peter Brusov, Tatiana Filatova, Mukhadin Eskindarov, Natali Orehova. 2012a. Influence of debt financing on the effectiveness of the finite duration investment project, Applied Financial Economics,V. 22, №13, P.1043-1052.

Brennan M., Schwartz E. 1978. Corporate Income Taxes, Valuation, and the Problem of Optimal Capital Structure // Journal of Business. P.51.

Leland H. 1994. Corporate Debt Value, Bond Covenants, and Optimal Capital Structure // Journal of Finance XLIX.

Brennan, M. J., and E. S. Schwartz. 1984. Optimal Financial Policy and Firm Valuation. Journal of Finance, 39, P. 593-607. http://dx.doi.org/10.1111/j.1540-6261.1984.tb03647.x

Kane, A., A. J. Marcus, and R. L. McDonald. 1984. How Big is the Tax Advantage to Debt? Journal of Finance, 39,841-853. http://dx.doi.org/10.2307/2327948

Dittmar A., Thakor A. 2007. Why Do Ffirms Issue Equity? // Journal of Finance.

Bikhchandani S., Hirshleifer D., Welch I. 1998. Learning From the Behavior of Jthers: Conformity, Fads, and Informational Cascades // The Journal of Economic Perspectives. Vol. 12. №3.

Post J.E., Preston L. E., Sachs S. 2002. Redefining the Corporation: Stakeholder Management and Organizational Wealth. Stanford: Stanford University Press.

Filbeck G., Raymond F., Preece D. 1996. Behavioral aspects of the intra-industry capital structure decision. Journal of Financial And Strategic Decisions, 9.

Jenter D. 2005. Market Timing and Managerial Portfolio Decisions // Journal of Finance. № 60 .

Baker M., Wurgler J. 2002. Market Timing and Capital Structure//Journal of Finance. - № 57. P.3.

Graham J., Harvey C. 2001. The Theory and Practice of Corporate Finance: Evidence from the Field // Journal of Financial Economics. №60.

Hovakimian A., Opler T., Titman S. 2001. The Debt-Equity Choice. Journal of Financial and Quantitative Analysis 36.

Myers S., Majluf N. 1984. Corporate Financing and Investment Decisions When Firms Have Information That Investors Do Not Have // Journal of Financial Economics. №13.

Myers S.C. 1984. The Capital Structure Pussle // Journal of Finance. July.

Hovakimian A., Opler T., Titman S. 2001. The Debt-Equity Choice. Journal of Financial and Quantitative Analysis 36.

Fama E., French K. 2004. Financing Decisions: Who Issues Stock? // Working Paper.

Jensen M. C., Meckling W. H. 1973. Theory of the Firm: Managerial Behavior, Agency Costs, and Ownership Structure // Journal of Financial Economics, V3. - № 5.

Brusov Peter, Filatova Tatiana, Orehova Natalia, Brusov Pavel, Brusova Nastia, 2011c. Influence of debt financing on the effectiveness of the investment project within the ModiglianiMiller theory, Research Journal of Economics, Business and ICT, v.2 (UK).

Brusov Peter, Filatova Tatiana, Mukhadin Eskindarov, Orehova Natalia, 2012b. Hidden global causes of the global financia crisis. Journal of Reviews on Global Economics, 1,106-111.

Received on 29-03-2013

Accepted on 10-04-2013

Published on 23-04-2013

\section{DOI: http://dx.doi.org/10.6000/1929-7092.2013.02.8}

(C) 2013 Brusov et al.; Licensee Lifescience Global.

This is an open access article licensed under the terms of the Creative Commons Attribution Non-Commercial License (http://creativecommons.org/licenses/by-nc/3.0/) which permits unrestricted, non-commercial use, distribution and reproduction in any medium, provided the work is properly cited. 